TRANSACTIONS OF THE

AMERICAN MATHEMATICAL SOCIETY

Volume 355, Number 6, Pages 2477-2500

S 0002-9947(03)03231-8

Article electronically published on January 14, 2003

\title{
REGULARITY OF WEAK SOLUTIONS TO THE MONGE-AMPÈRE EQUATION
}

\author{
CRISTIAN E. GUTIÉRREZ AND DAVID HARTENSTINE
}

\begin{abstract}
We study the properties of generalized solutions to the MongeAmpère equation $\operatorname{det} D^{2} u=\nu$, where the Borel measure $\nu$ satisfies a condition, introduced by Jerison, that is weaker than the doubling property. When $\nu=f d x$, this condition, which we call $D_{\epsilon}$, admits the possibility of $f$ vanishing or becoming infinite. Our analysis extends the regularity theory (due to Caffarelli) available when $0<\lambda \leq f \leq \Lambda<\infty$, which implies that $\nu=f d x$ is doubling. The main difference between the $D_{\epsilon}$ case and the case when $f$ is bounded between two positive constants is the need to use a variant of the Aleksandrov maximum principle (due to Jerison) and some tools from convex geometry, in particular the Hausdorff metric.
\end{abstract}

\section{INTRODUCTION}

In this paper, we present some results that are extensions of the regularity theory for generalized solutions of the Monge-Ampère equation det $D^{2} u=f$, where $0<$ $\lambda \leq f \leq \Lambda<\infty$, developed by Caffarelli, C90, [C91. We shall assume that the right-hand side $f$ satisfies a condition, weaker than the doubling property (and therefore weaker than $\lambda \leq f \leq \Lambda$ ), that was introduced by $D$. Jerison in a very interesting paper J96 in connection with the Minkoswki problem for electrostatic capacity. We shall call this condition $D_{\epsilon}$, see Definition 2.6. Jerison claimed that strictly convex solutions to $\operatorname{det} D^{2} u=f$ with $f$ satisfying $D_{\epsilon}$ and $u=0$ on the boundary are $C^{1, \alpha}$; see [J96, Theorem 7.1]. As a tool, he generalizes a maximum principle due to Aleksandrov, Theorem [2.8, but the proof of the $C^{1, \alpha}$ estimates is not included in his paper.

Our purpose in this paper is to elaborate on the condition $D_{\epsilon}$. We shall first prove that for global convex functions $u$ in $\mathbb{R}^{n}, D_{\epsilon}$ and doubling coincide, Theorem 3.1. To this end we use a geometric characterization of the doubling property of Monge-Ampère measures given in GH00. We next prove under $D_{\epsilon}$ an extremal points theorem and a selection lemma having independent interest. These two results are the main pillars in Caffarelli's theory as presented in G01, Chapter 5]. Once these results are established, we obtain strict convexity of generalized solutions, and as a consequence we prove interior $C^{1, \alpha}$ estimates. Unlike the case when $f$ is bounded between two constants, under $D_{\epsilon}$ we need to use the Hausdorff

Received by the editors March 3, 2002 and, in revised form, October 7, 2002.

2000 Mathematics Subject Classification. Primary 35D10, 35J65, 35J60.

Key words and phrases. Aleksandrov solutions, strict convexity, Hausdorff metric, doubling property, Hölder estimates.

The first author was partially supported by NSF grant DMS-0070648. 
metric to establish some of the theorems. Our work clarifies some of the claims made by Jerison and presents the details of the argument.

The paper is organized as follows. Section 2 contains definitions and preliminary results. The proof that for global convex functions $D_{\epsilon}=D_{1}$ is contained in Section 3 Section 4 contains the extremal points theorem and as a consequence the strict convexity, Corollary 4.2 In Section 5 we state and prove some facts concerning Hausdorff convergence that are needed to prove the selection result in Section 6 Finally, combining the results in the previous sections, we prove in Section 7 the $C^{1, \alpha}$ estimates.

\section{Preliminaries}

We begin this section by reviewing some of the basic theory of the MongeAmpère equation.

Given $u: \Omega \rightarrow \mathbb{R}$, we recall that the normal mapping of $u$ is defined by

$$
\partial u\left(x_{0}\right)=\left\{p \in \mathbb{R}^{n}: u(x) \geq u\left(x_{0}\right)+p \cdot\left(x-x_{0}\right), \forall x \in \Omega\right\} ;
$$

and if $E \subset \Omega$, then we set $\partial u(E)=\bigcup_{x \in E} \partial u(x)$. Note that the normal map of $u$ at a point $x_{0}$ is the set of points $p$ that determine supporting hyperplanes to $u$ at $x_{0}$.

If $\Omega$ is open and $u \in C(\Omega)$, then the family of sets

$$
S=\{E \subset \Omega: \partial u(E) \text { is Lebesgue measurable }\}
$$

is a Borel $\sigma$-algebra. The map $M u: S \rightarrow \overline{\mathbb{R}}$ defined by $M u(E)=|\partial u(E)|$ (where $|S|$ indicates the Lebesgue measure of the set $S$ ) is a measure, finite on compact subsets, called the Monge-Ampère measure associated with the function $u$. The convex function $u$ is a weak (Aleksandrov) solution of $\operatorname{det} D^{2} u=\nu$ if the MongeAmpère measure $M u$ associated with the function $u$ equals the Borel measure $\nu$.

The following lemma will be used to prove the convergence results below. This is [G01, Lemma 1.2.3]. In fact, this is a special case of a more general result on weak solutions of $k$-Hessian equations, see [TW97, Theorem 1.1].

Lemma 2.1. If $u_{k}$ is a sequence of convex functions in $\Omega$ such that $u_{k} \rightarrow u$ uniformly on compact subsets of $\Omega$, then $M u_{k} \rightarrow M u$ weakly, meaning that

$$
\int_{\Omega} f(x) d M u_{k}(x) \rightarrow \int_{\Omega} f(x) d M u(x)
$$

for every continuous function $f$ with compact support in $\Omega$.

In considering the regularity properties of solutions, it is convenient to analyze the properties of the following sets.

Definition 2.2. Let $u: \Omega \rightarrow \mathbb{R}$ be convex. The cross-sections of $u$ are the (convex) sets

$$
S\left(x_{0}, p, t\right)=\left\{x \in \Omega: u(x)<u\left(x_{0}\right)+p \cdot\left(x-x_{0}\right)+t\right\},
$$

where $p \in \partial u\left(x_{0}\right)$ and $t>0$.

The ability to transfer our analysis from a general convex set to a normalized setting is fundamental to what follows. The theorem below is what allows us to do this.

Definition 2.3. A convex set $\Omega$ is said to be normalized if its center of mass $c(\Omega)=0$ and $B_{\alpha_{n}}(0) \subset \Omega \subset B_{1}(0)$, where $\alpha_{n}$ is a dimensional constant. 
Theorem 2.4 (F. John). Let $\Omega \subset \mathbb{R}^{n}$ be open, bounded, and convex.

(a) Out of all ellipsoids that contain $\Omega$, there exists one of minimal volume.

(b) There exists an invertible affine transformation $T$ such that $T(\Omega)$ is normalized.

Following Jerison [J96, p. 31], we define a dimensionless, normalized distance invariant under affine transformations.

Definition 2.5. The normalized distance from $x \in \bar{S}$ to the boundary of the convex set $S$ is

$$
\delta(x, S)=\min \left\{\frac{\left|x-x_{1}\right|}{\left|x-x_{2}\right|}: x_{1}, x_{2} \in \partial S \text { and } x, x_{1}, x_{2} \text { are collinear }\right\} .
$$

If $T$ is an affine transformation, then $\delta(x, S)=\delta(T x, T(S))$; also, if the set $S$ is normalized, then $\delta(x, S) \approx \operatorname{dist}(x, \partial S)$. This distance is continuous in $x$, and note that $\delta(x, S) \leq 1$. For $\alpha>0$ and $S$ convex we define $\alpha S$ to be the $\alpha$-dilation of $S$ with respect to its standard center of mass.

We are now in a position to define the $D_{\epsilon}$ condition.

Definition 2.6. Let $0<\epsilon \leq 1, \mu$ a Borel measure in $\Omega$, and $u$ convex in $\Omega$. The measure $\mu$ is $D_{\epsilon}$ in $\Omega$ on the sections of $u$, or $\mu \in D_{\epsilon}$, if

$$
\int_{S} \delta(x, S)^{1-\epsilon} d \mu \leq C \mu\left(\frac{1}{2} S\right)
$$

for all sections $S$ of $u$ compactly contained in $\Omega$, that is, $S \Subset \Omega$. By $D_{\epsilon}(C)$ we denote the measures satisfying $D_{\epsilon}$ with constant $C$.

Notice that this family of conditions includes the doubling condition $\mu(S) \leq$ $C \mu\left(\frac{1}{2} S\right)$, which corresponds to $\epsilon=1$. Since $\delta(x, S) \leq 1$, if $\mu \in D_{\epsilon_{0}}$, then $\mu \in D_{\epsilon}$ for all $\epsilon<\epsilon_{0}$. In particular, if $\mu$ is doubling, then it is $D_{\epsilon}$ for all $\epsilon$. In the case $M u=\mu=f d x$, this condition allows for the possibility of $f$ to vanish or become infinite. This condition also has an extension to the entire domain under the additional hypothesis that $u \in C(\bar{\Omega})$ and vanishes on the boundary. See Lemma 5.9 below.

Remark 2.7. We next derive a formula for an affine change of variables, see [G01, p. 47]. Let $T$ be an invertible affine transformation, $T x=A x+b$ for some nonsingular matrix $A$ and some $b \in \mathbb{R}^{n}$. Suppose $u: \Omega \rightarrow \mathbb{R}$ and $v(y)=\lambda^{-1} u\left(T^{-1} y\right)$ where $\lambda>0$. The affine function $l(x)=u\left(x_{0}\right)+p \cdot\left(x-x_{0}\right)$ is a supporting hyperplane to $u$ at $x_{0}$ if and only if $\bar{l}(y)=v\left(T x_{0}\right)+\lambda^{-1}\left(A^{-1}\right)^{t} p \cdot\left(y-T x_{0}\right)$ is a supporting hyperplane to $v$ at $T x_{0}$. This means that if $S=S_{u}\left(x_{0}, p, t\right)$ is a section of $u$, then $T(S)$ is a section of $v$. More precisely, $T(S)=S_{v}\left(T x_{0}, \lambda^{-1}\left(A^{-1}\right)^{t} p, \frac{t}{\lambda}\right)$. This also implies that

and hence that

$$
\frac{1}{\lambda}\left(A^{-1}\right)^{t}(\partial u(E))=\partial v(T E)
$$

$$
M v(T E)=\frac{1}{\lambda^{n}}\left|\operatorname{det} A^{-1}\right| M u(E)
$$

for any Borel set $E \subset \Omega$. From this formula and the fact that, for any section $S$, $T(\alpha S)=\alpha T(S)$, we get that if $M u$ is $D_{\epsilon}$ in $\Omega$, then $M v$ is $D_{\epsilon}$ in $T(\Omega)$, with the same constant. 
The following theorem is an extension of a classical maximum principle due to Aleksandrov, G01, Theorem 1.4.2]. The advantage of this generalization is that one can deal, for example, with convex functions $u$ with $M u(\Omega)=+\infty$ for which the Aleksandrov estimate does not give information.

Theorem 2.8 ([J96, Lemma 7.3]). If $\Omega$ is convex and normalized, $u \in C(\bar{\Omega})$ is convex, $\left.u\right|_{\partial \Omega}=0$, and $0<\epsilon \leq 1$, then

$$
\left|u\left(x_{0}\right)\right|^{n} \leq C(n, \epsilon) \delta\left(x_{0}, \Omega\right)^{\epsilon} \int_{\Omega} \delta(x, \Omega)^{1-\epsilon} d M u
$$

for all $x_{0} \in \Omega$.

We recall the following lemma.

Lemma 2.9 ([G01, Lemma 3.2.1]). Let $\Omega \subset \mathbb{R}^{n}$ be bounded, convex and open, and $\phi$ a convex function in $\Omega$ such that $\phi \leq 0$ on $\partial \Omega$. If $x \in \Omega$ and $l(y)=\phi(x)+p \cdot(y-x)$ is a supporting hyperplane to $\phi$ at the point $(x, \phi(x))$, then

$$
|p| \leq \frac{-\phi(x)}{\operatorname{dist}(x, \partial \Omega)} .
$$

More generally, if $\bar{\Omega}_{0} \subset \Omega$, then

$$
\partial \phi\left(\Omega_{0}\right) \subset B\left(0, \frac{\max _{\Omega_{0}}(-\phi)}{\operatorname{dist}\left(\Omega_{0}, \partial \Omega\right)}\right) .
$$

As a first consequence of Theorem 2.8, we obtain the following equivalence, which will be important later.

Proposition 2.10. Let $\Omega$ be open, convex, and normalized, $u \in C(\bar{\Omega})$, convex and $\left.u\right|_{\partial \Omega}=0$. Suppose

$$
\int_{\Omega} \delta(x, \Omega)^{1-\epsilon} d M u \leq C M u\left(\frac{1}{2} \Omega\right) .
$$

Then there exist two constants $C_{1}=C_{1}(n, \epsilon)$ and $C_{2}=C_{2}(n, \epsilon, C)$ such that

$$
C_{1}\left|\min _{\Omega} u\right|^{n} \leq \int_{\Omega} \delta(x, \Omega)^{1-\epsilon} d M u \leq C_{2}\left|\min _{\Omega} u\right|^{n} .
$$

Proof. The first inequality follows directly from Theorem 2.8 and the fact that $\delta(x, \Omega) \leq 1$. In fact, for this inequality the hypothesis concerning the integral is not needed. For the second inequality, we have

$$
\int_{\Omega} \delta(x, \Omega)^{1-\epsilon} d M u \leq C M u\left(\frac{1}{2} \Omega\right) \leq \tilde{C}\left|\min _{\Omega} u\right|^{n},
$$

where the second inequality is a consequence of the preceding lemma and the fact that since $\Omega$ is normalized, $\operatorname{dist}\left(\frac{1}{2} \Omega, \partial \Omega\right) \geq C(n)$.

\section{Comparison of $D_{1}$ And $D_{\epsilon}$}

In this section, we show that if the convex function $u$ is defined on all of $\mathbb{R}^{n}$ and $M u \in D_{\epsilon}$, then $M u$ is doubling. Because the doubling condition implies $D_{\epsilon}$ for every $\epsilon>0$, this means that for globally defined functions, the two conditions are equivalent. We also provide an example to show that this is not true on bounded domains.

Theorem 3.1. If $u: \mathbb{R}^{n} \rightarrow \mathbb{R}$ is convex and $M u \in D_{\epsilon}$ for some $\epsilon \in(0,1)$, then $M u$ is doubling. 
The proof of this result uses the following characterization of doubling MongeAmpère measures on $\mathbb{R}^{n}$, due to Gutiérrez and Huang [GH00]:

Theorem 3.2 ([G01, Theorem 3.3.5]). $M u=\mu$ is doubling on $\mathbb{R}^{n}$ if and only if there exist constants $0<\tau, \lambda<1$, such that for all $x_{0}$ and $t>0, S\left(x_{0}, p, \tau t\right) \subset$ $\lambda S\left(x_{0}, p, t\right)$.

Proof of Theorem 3.1. Let $S=S_{u}\left(x_{0}, p, t\right)$ be any section of $u$. Let $T$ be an affine transformation that normalizes $S, T x=A x+b$ for an invertible matrix $A$, and denote $T(S)$ by $S^{*}$. Define $v(x)=u\left(T^{-1} x\right)$. Then $T\left(S_{u}\left(x_{0}, p, \lambda t\right)\right)=S_{v}\left(T x_{0}, q, \lambda t\right)$ for any $\lambda>0$, where $q=\left(A^{-1}\right)^{t} p$. We also have $T(\lambda S)=\lambda T(S)=\lambda S^{*}$.

Let $v^{*}(x)=v(x)-v\left(T x_{0}\right)-q \cdot\left(x-T x_{0}\right)-t$. Then $\partial v^{*}=\partial v-q$, and by the translation invariance of Lebesgue measure $M v^{*}=M v$. Also, $\left.v^{*}\right|_{\partial S^{*}}=0$. Let $y \in S^{*} \backslash \lambda S^{*}$, where $\lambda<1$ is to be chosen, close to 1 .

Since $S^{*}$ is normalized, $\operatorname{dist}\left(y, \partial S^{*}\right) \leq(1-\lambda)$ implies that $\delta\left(y, S^{*}\right) \leq C_{n}(1-\lambda)$. Then, by Theorem 2.8 and Proposition 2.10 .

$$
\begin{aligned}
\left|v^{*}(y)\right|^{n} & \leq C \delta\left(y, S^{*}\right)^{\epsilon} \int_{S^{*}} \delta\left(x, S^{*}\right)^{1-\epsilon} d M v^{*} \\
& \leq C(1-\lambda)^{\epsilon}\left|\min _{S^{*}} v^{*}\right|^{n} \\
& =C(1-\lambda)^{\epsilon} t^{n} .
\end{aligned}
$$

Therefore $v^{*}(y) \geq-C(1-\lambda)^{\epsilon / n} t$, meaning that

$$
v(y)-v\left(T x_{0}\right)-q \cdot\left(y-T x_{0}\right) \geq\left(1-C(1-\lambda)^{\epsilon / n}\right) t .
$$

Choose $\lambda$ so that the term on the right-hand side is positive. Then choose $0<\tau<1-C(1-\lambda)^{\epsilon / n}$.

Therefore,

$$
v(y) \geq v\left(T x_{0}\right)+q \cdot\left(y-T x_{0}\right)+\tau t ;
$$

so $y \notin S_{v}\left(T x_{0}, q, \tau t\right)$.

This implies that $S_{v}\left(T x_{0}, q, \tau t\right) \subset \lambda S^{*}$. By applying $T^{-1}$, we get the inclusion $S_{u}\left(x_{0}, p, \tau t\right) \subset \lambda S_{u}\left(x_{0}, p, t\right)$. So by Theorem 3.2, $M u$ is doubling.

The following example shows that $D_{\epsilon}$ is not equivalent to $D_{1}$ on bounded domains.

Define the function $u:[0,1] \rightarrow \mathbb{R}$ by

$$
u(x)= \begin{cases}x \log x, & 0<x \leq 1 \\ 0, & x=0\end{cases}
$$

This function is continuous on $[0,1]$, convex, and satisfies $M u=\frac{1}{x} d x$.

Since $u$ is zero on the boundary (i.e., $u(0)=u(1)=0$ ), there are sections of the form $(a, b)$ where $0<a$ can be arbitrarily small, and $\frac{9}{10}<b<1$. Then $M u((a, b))=\ln \left(\frac{b}{a}\right)$. Since $a$ can be chosen as small as we like, sections can have arbitrarily large $M u$ measure. However, for all such sections (if $a<\frac{1}{10}$ ), we have that $\frac{1}{2}(a, b) \subset\left(\frac{9}{40}, \frac{31}{40}\right)$, so that $M u\left(\left(\frac{1}{2}(a, b)\right) \leq \ln \left(\frac{31}{9}\right)\right.$. Therefore, the measure $M u$ is not doubling.

In this one-dimensional setting, all sections are intervals and $\delta(x, S)$ can be calculated explicitly for any section. Some elementary calculations show that $M u$ satisfies the $D_{\frac{1}{2}}$ condition. 


\section{Extremal Points and Strict Convexity}

In this section, we prove a Caffarelli-type extremal points theorem for convex functions $u$ satisfying $M u \in D_{\epsilon}$. For basic results of convex geometry we refer to Schneider [Sc93].

Theorem 4.1. Let $\Omega \subset \mathbb{R}^{n}$ be open, bounded and convex, and let $u \in C(\Omega)$ be convex. Suppose $M u \in D_{\epsilon}$. Assume $u \geq 0$ and let $\Gamma=\{x \in \Omega: u(x)=0\}$. If $\Gamma$ contains more than one point, then $\Gamma$ has no extremal points in $\Omega$.

Proof. The argument follows Caffarelli's construction given in [G01, Theorem 5.2.1] for the case when $M u$ is bounded between two constants. The main differences between that proof and the argument here are the use of the Aleksandrov-Jerison estimate (Theorem 2.8) instead of the classical version, and the need for more care concerning the measure since the $D_{\epsilon}$ condition applies only to sections and not general measurable sets.

Suppose, for a contradiction, that $x_{0} \in \Omega$ is an extremal point of $\Gamma$. Applying G01, Lemma 5.1.4] to the set $\Gamma$, we have that given $\delta>0$ there exist a supporting hyperplane $l(x)$ at some point of $\partial \Gamma$ (not necessarily $x_{0}$ ) and an $\epsilon_{0}>0$ such that

(a) $\Gamma \subset\{x: l(x) \geq 0\}$,

(b) $\operatorname{diam}\left\{x \in \bar{\Gamma}: 0 \leq l(x) \leq \epsilon_{0}\right\}<\delta$, and

(c) $0 \leq l\left(x_{0}\right)<\epsilon_{0}$.

We take $\delta=\rho<\frac{1}{2} \operatorname{dist}\left(x_{0}, \partial \Omega\right)$, and let $x_{1}$ be the point at which $l(x)$ is a supporting hyperplane.

Define

$$
\begin{aligned}
S & =\left\{x \in \Gamma: 0 \leq l(x) \leq \epsilon_{0}\right\}, \\
\Pi_{1} & =\left\{x: l(x)=\epsilon_{0}\right\}, \\
\Pi_{2} & =\{x: l(x)=0\},
\end{aligned}
$$

and for $\epsilon_{1}>0$, the convex set

$$
\Gamma_{\epsilon_{1}}=\left\{x \in \Omega: u(x) \leq \epsilon_{1}\left(\epsilon_{0}-l(x)\right)\right\} .
$$

We have that $S=\bigcap_{\epsilon_{1}>0} \Gamma_{\epsilon_{1}}$, and by the choice of $\delta, \Gamma_{\epsilon_{1}} \subset \Omega^{\circ}$ for all $\epsilon_{1}$ sufficiently small. Also, $\Gamma_{\epsilon_{1}}$ is the closure of a section of $u$ in $\Omega$. Now slide $\Pi_{2}$ in a parallel fashion away from $\Pi_{1}$ until it touches $\partial \Gamma_{\epsilon_{1}}$ at a point $x_{\epsilon_{1}}$ and let $\Pi_{3}$ denote the resulting plane, i.e.,

$$
\Pi_{3}=\left\{x: l(x)=-\rho_{\epsilon_{1}}\right\}, \quad x_{\epsilon_{1}} \in \Pi_{3}, \quad \Gamma_{\epsilon_{1}} \subset\left\{x:-\rho_{\epsilon_{1}} \leq l(x) \leq \epsilon_{0}\right\},
$$

with $\rho_{\epsilon_{1}}>0$.

Let $u_{\epsilon_{1}}(x)=u(x)-\epsilon_{1}\left(\epsilon_{0}-l(x)\right)$. We have

(a) $\inf _{\Gamma_{\epsilon_{1}}} u_{\epsilon_{1}}<0$,

(b) $\Gamma_{\epsilon_{1}}$ is a section of $u_{\epsilon_{1}}$,

(c) $u_{\epsilon_{1}} \mid \partial \Gamma_{\epsilon_{1}}=0$,

(d) $\frac{\operatorname{dist}\left(\Pi_{2}, \Pi_{3}\right)}{\operatorname{dist}\left(\Pi_{1}, \Pi_{2}\right)}=\frac{\rho_{\epsilon_{1}}}{\epsilon_{0}} \rightarrow 0$ as $\epsilon_{1} \rightarrow 0 ;$

and consequently,

$$
\liminf _{\epsilon_{1} \rightarrow 0} \frac{\left|u_{\epsilon_{1}}\left(x_{1}\right)\right|}{\left|\inf _{\Gamma_{\epsilon_{1}}} u_{\epsilon_{1}}\right|} \geq 1 .
$$


Let $T_{\epsilon_{1}}$ normalize $\Gamma_{\epsilon_{1}}$ and $u_{\epsilon_{1}}^{*}(x)=u_{\epsilon_{1}}\left(T_{\epsilon_{1}}^{-1} x\right)$. Then $M u_{\epsilon_{1}}^{*} \in D_{\epsilon}$ and $u_{\epsilon_{1}}^{*}$ is zero on $\partial \Gamma_{\epsilon_{1}}^{*}$ where $\Gamma_{\epsilon_{1}}^{*}=T_{\epsilon_{1}}\left(\Gamma_{\epsilon_{1}}\right)$. Then, as above,

$$
\frac{\left|u_{\epsilon_{1}}^{*}\left(T_{\epsilon_{1}} x_{1}\right)\right|}{\left|\inf _{\Gamma_{\epsilon_{1}}^{*}} u_{\epsilon_{1}}^{*}\right|} \geq C_{1}>0
$$

for $\epsilon_{1}$ sufficiently small. Proposition 2.10 applied to $u_{\epsilon_{1}}^{*}$ on the set $\Gamma_{\epsilon_{1}}^{*}$ then yields

$$
\left|u_{\epsilon_{1}}^{*}\left(T_{\epsilon_{1}} x_{1}\right)\right|^{n} \geq C_{1}^{n}\left|\inf _{\Gamma_{\epsilon_{1}}^{*}} u_{\epsilon_{1}}^{*}\right|^{n} \geq C \int_{\Gamma_{\epsilon_{1}^{*}}^{*}} \delta\left(x, \Gamma_{\epsilon_{1}}^{*}\right)^{1-\epsilon} d M u_{\epsilon_{1}}^{*} .
$$

To see that we can apply Proposition 2.10 here, we first see that using (2.1) we have

$$
\begin{aligned}
M u_{\epsilon_{1}}^{*}\left(\frac{1}{2} \Gamma_{\epsilon_{1}}^{*}\right) & =M u_{\epsilon_{1}}^{*}\left(\frac{1}{2} T_{\epsilon_{1}}\left(\Gamma_{\epsilon_{1}}\right)\right)=M u_{\epsilon_{1}}^{*}\left(T_{\epsilon_{1}}\left(\frac{1}{2} \Gamma_{\epsilon_{1}}\right)\right) \\
& =\left|\operatorname{det} T_{\epsilon_{1}}^{-1}\right| M u_{\epsilon_{1}}\left(\frac{1}{2} \Gamma_{\epsilon_{1}}\right)=\left|\operatorname{det} T_{\epsilon_{1}}^{-1}\right| M u\left(\frac{1}{2} \Gamma_{\epsilon_{1}}\right) .
\end{aligned}
$$

Secondly, again by 2.1) and the invariance of $\delta$ under affine transformations, we get

$$
\int_{\Gamma_{\epsilon_{1}}^{*}} \delta\left(x, \Gamma_{\epsilon_{1}}^{*}\right)^{1-\epsilon} d M u_{\epsilon_{1}}^{*}=\int_{\Gamma_{\epsilon_{1}}} \delta\left(y, \Gamma_{\epsilon_{1}}\right)^{1-\epsilon}\left|\operatorname{det} T_{\epsilon_{1}}^{-1}\right| d M u .
$$

Since $\Gamma_{\epsilon_{1}}$ is a section of $u$ and $M u \in D_{\epsilon}$,

$$
\int_{\Gamma_{\epsilon_{1}}} \delta\left(y, \Gamma_{\epsilon_{1}}\right)^{1-\epsilon} d M u \leq C M u\left(\frac{1}{2} \Gamma_{\epsilon_{1}}\right) .
$$

Therefore, by canceling the factor $\left|\operatorname{det} T_{\epsilon_{1}}^{-1}\right|$ we get the inequality

$$
\int_{\Gamma_{\epsilon_{1}}^{*}} \delta\left(x, \Gamma_{\epsilon_{1}}^{*}\right)^{1-\epsilon} d M u_{\epsilon_{1}}^{*} \leq C M u_{\epsilon_{1}}^{*}\left(\frac{1}{2} \Gamma_{\epsilon_{1}}^{*}\right),
$$

which is what we need to apply Proposition 2.10, so (4.1) holds.

The next step is to show that

$$
\operatorname{dist}\left(T_{\epsilon_{1}} x_{1}, \partial \Gamma_{\epsilon_{1}}^{*}\right) \rightarrow 0, \quad \text { as } \epsilon_{1} \rightarrow 0 .
$$

Let $\Pi_{i}^{*}$ denote $T_{\epsilon_{1}} \Pi_{i}$ for $i=1,2$ and 3 . We first prove that as $\epsilon_{1} \rightarrow 0$,

$$
\frac{\operatorname{dist}\left(\Pi_{2}^{*}, \Pi_{3}^{*}\right)}{\operatorname{dist}\left(\Pi_{1}^{*}, \Pi_{2}^{*}\right)} \rightarrow 0
$$

We have that $\operatorname{dist}\left(\Pi_{1}^{*}, \Pi_{2}^{*}\right) \leq \operatorname{dist}\left(\Pi_{1}^{*} \cap \Gamma_{\epsilon_{1}}^{*}, \Pi_{2}^{*} \cap \Gamma_{\epsilon_{1}}^{*}\right) \leq 2$, since $\Gamma_{\epsilon_{1}}^{*}$ is normalized. Then

$$
\frac{\operatorname{dist}\left(\Pi_{2}^{*}, \Pi_{3}^{*}\right)}{\operatorname{dist}\left(\Pi_{1}^{*}, \Pi_{2}^{*}\right)}=\frac{\operatorname{dist}\left(\Pi_{2}, \Pi_{3}\right)}{\operatorname{dist}\left(\Pi_{1}, \Pi_{2}\right)}=\frac{\rho_{\epsilon_{1}}}{\epsilon_{0}} \rightarrow 0
$$

as $\epsilon_{1} \rightarrow 0$, and hence $\operatorname{dist}\left(\Pi_{2}^{*}, \Pi_{3}^{*}\right) \rightarrow 0$. Now let $\partial \Gamma_{2}^{*} \subset \partial \Gamma_{\epsilon_{1}}^{*}$ be that portion of the boundary lying between the planes $\Pi_{2}^{*}$ and $\Pi_{3}^{*}$. Let $P_{\epsilon_{1}} \in \partial \Gamma_{2}^{*}$ be the point such that the line through $T_{\epsilon_{1}} x_{1} \in \Pi_{2}^{*}$ and $P_{\epsilon_{1}}$ is normal to $\Pi_{2}^{*}$. Then $\operatorname{dist}\left(T_{\epsilon_{1}} x_{1}, \partial \Gamma_{\epsilon_{1}}^{*}\right) \leq \operatorname{dist}\left(T_{\epsilon_{1}} x_{1}, \partial \Gamma_{2}^{*}\right) \leq\left|T_{\epsilon_{1}} x_{1}-P_{\epsilon_{1}}\right| \leq \operatorname{dist}\left(\Pi_{2}^{*}, \Pi_{3}^{*}\right) \rightarrow 0$.

On the other hand, by Theorem 2.8

$$
\left|u_{\epsilon_{1}}^{*}\left(T_{\epsilon_{1}} x_{1}\right)\right|^{n} \leq C \delta\left(T_{\epsilon_{1}} x_{1}, \Gamma_{\epsilon_{1}}^{*}\right)^{\epsilon} \int_{\Gamma_{\epsilon_{1}}^{*}} \delta\left(x, \Gamma_{\epsilon_{1}}^{*}\right)^{1-\epsilon} d M u_{\epsilon_{1}}^{*}
$$


From (4.1) above,

$$
\left|u_{\epsilon_{1}}^{*}\left(T_{\epsilon_{1}} x_{1}\right)\right|^{n} \geq C \int_{\Gamma_{\epsilon_{1}}^{*}} \delta\left(x, \Gamma_{\epsilon_{1}}^{*}\right)^{1-\epsilon} d M u_{\epsilon_{1}}^{*} .
$$

This implies that $\delta\left(T_{\epsilon_{1}} x_{1}, \Gamma_{\epsilon_{1}}^{*}\right)^{\epsilon} \geq C$. However, since $\Gamma_{\epsilon_{1}}^{*}$ is normalized,

$$
\delta\left(T_{\epsilon_{1}} x_{1}, \Gamma_{\epsilon_{1}}^{*}\right) \approx \operatorname{dist}\left(T_{\epsilon_{1}} x_{1}, \partial \Gamma_{\epsilon_{1}}^{*}\right) .
$$

This contradicts (4.2), and the proof is complete.

Corollary 4.2. Let $\Omega \subset \mathbb{R}^{n}$ be open, bounded and convex. Suppose $u \in C(\bar{\Omega})$ is convex and $u=0$ on $\partial \Omega$. Then if $M u \in D_{\epsilon}$, $u$ is either strictly convex or identically zero.

Proof. Suppose $u$ is not strictly convex. Then the graph of $u$ contains a line segment, say $L$. If $\left(x_{0}, u\left(x_{0}\right)\right) \in \operatorname{int}(L)$, then any supporting hyperplane $l(x)$ to $u$ at $x_{0}$ must contain $L$.

Apply Theorem 4.1 to the function $u(x)-l(x)$. This function is nonnegative on $\Omega$, and the set $\Gamma=\{x \in \Omega: u(x)=l(x)\}$ contains more than one point. Therefore, $\Gamma$ has no extremal points inside $\Omega$. So all of its extremal points are in $\partial \Omega$.

Write $x_{0}=\sum_{i=1}^{m} \lambda_{i} x_{i}$, where the $x_{i} \in \partial \Omega$ are extremal points of $\Gamma, \lambda_{i}>0$, and $\sum \lambda_{i}=1$. Then $u\left(x_{0}\right)=l\left(x_{0}\right)=\sum \lambda_{i} l\left(x_{i}\right)=\sum \lambda_{i} u\left(x_{i}\right)=0$, since $u\left(x_{i}\right)=0$. Then, because $x_{0}$ is an interior point of $\Omega$ and $u\left(x_{0}\right)=0, u \equiv 0$ by convexity.

Now that the extremal points theorem has been established for $D_{\epsilon}$, we may use the same barrier argument as in [G01, Theorem 5.4.7] to deduce the strict convexity of functions with nonhomogeneous boundary values. We remark that this theorem is sharp. See [P78, pp. 81-84] for examples.

Theorem 4.3. Let $\Omega$ be bounded, open and convex, and let $u \in C(\bar{\Omega})$ be convex. Suppose $M u \in D_{\epsilon}$ for some $\epsilon$, and $M u>\lambda>0$. Then if $u=f$ on $\partial \Omega$, where $f \in C^{1, \beta}(\partial \Omega)$ for $\beta>1-\frac{2}{n}(n \geq 3)$, then $u$ is strictly convex.

\section{Some Results about the Hausdorff Metric}

In this section we collect the results about Hausdorff convergence needed for the proof of Lemma 6.1.

Let $K^{n}$ denote the set of nonempty compact subsets of $\mathbb{R}^{n}$. In the rest of this paper, whenever we refer to the convergence of sets, we mean convergence with respect to the following metric.

Definition 5.1. For $K, L \in K^{n}$, the Hausdorff metric is defined by

$$
d_{H}(K, L)=\max \left\{\max _{x \in K} \min _{y \in L}|x-y|, \max _{x \in L} \min _{y \in K}|x-y|\right\},
$$

or equivalently by

$$
d_{H}(K, L)=\min \left\{\lambda \geq 0: K \subset L+\lambda B_{1}(0), L \subset K+\lambda B_{1}(0)\right\} .
$$

We also define the Minkowski support function of a closed convex set $K$.

Definition 5.2. Let $K \subset \mathbb{R}^{n}$ be closed, nonempty and convex. The Minkowski support function is the map $h(K, \cdot): \mathbb{R}^{n} \rightarrow \overline{\mathbb{R}}$ given by

$$
h(K, u)=\sup _{x \in K}\langle x, u\rangle .
$$


We quote the following result, establishing the connection between the Hausdorff metric and the support function. A convex body is a convex set in $K^{n}$.

Theorem 5.3 ([Sc93, Theorem 1.8.11]). For any convex bodies $K$ and $L$,

$$
d_{H}(K, L)=\sup _{u \in S^{n-1}}|h(K, u)-h(L, u)|
$$

We now collect some properties of the Hausdorff metric that will be used in the next section. First we present the following theorem, due to Blaschke, see Sc93. Theorem 1.8.6].

Theorem 5.4 (Blaschke Selection Theorem). From each bounded sequence of convex bodies, one can select a subsequence converging in the Hausdorff metric to a convex body.

The next lemma connects convergence in the Hausdorff metric with pointwise convergence of characteristic functions.

Lemma 5.5. Suppose $\left\{K_{n}\right\}$, a sequence of compact convex sets, converges to $K$. Then $\chi_{K_{n}}(x) \rightarrow \chi_{K}(x)$ pointwise for every $x \notin \partial K$.

Proof. By Theorem 5.4, $K$ is compact and convex. If $x \notin K$, then $x \notin K_{n}$ for $n$ sufficiently large, since $K_{n} \rightarrow K$.

Suppose $x \in K^{\circ}$, and let $0<\rho<\operatorname{dist}(x, \partial K)$. Then $B_{\rho}(x) \subset K$. Change the coordinates by a translation so that in the new coordinates $x=0$. From now on, $K_{n}$ and $K$ represent the translations of the original sets in question. For any $v \in S^{n-1}, K$ is contained between the parallel planes

$$
\left\{z \in \mathbb{R}^{n}:\langle z, v\rangle=h(K, v)\right\} \quad \text { and } \quad\left\{z \in \mathbb{R}^{n}:\langle z, v\rangle=h(K,-v)\right\} .
$$

Since $B_{\rho}(0) \subset K, h(K, v) \geq \rho$ for all $v \in S^{n-1}$. For $n \geq N(\rho)$, we have that $\left|h\left(u, K_{n}\right)-h(u, K)\right| \leq \frac{\rho}{2}$ for all $u \in S^{n-1}$. Therefore, $h\left(u, K_{n}\right) \geq \frac{\rho}{2}$ for any unit vector $u$, so that $B_{\frac{\rho}{2}}(0) \subset K_{n}$ for any $n$ large enough. This shows that $0 \in K_{n}^{\circ}$ for such $n$, and translating back to the original coordinates, we get that $x \in K_{n}^{\circ}$ for all $n \geq N$ for some $N$, proving the claim.

We now prove that if a sequence of convex bodies $\left\{K_{n}\right\}$ converges to $K$, then the sequence $\left\{\frac{1}{2} K_{n}\right\}$ converges to $\frac{1}{2} K$.

Lemma 5.6. If $K_{n} \rightarrow K$, then $\frac{1}{2} K_{n} \rightarrow \frac{1}{2} K$, where $\frac{1}{2} K_{n}$ is the dilation of $K_{n}$ (and similarly for $\left.\frac{1}{2} K\right)$ with respect to its center of mass $c\left(K_{n}\right)$.

Proof. We have the following formulas:

$$
\begin{gathered}
\frac{1}{2} K_{n}=\left\{\frac{1}{2}\left(c\left(K_{n}\right)+y\right): y \in K_{n}\right\} \\
{\left[c\left(K_{n}\right)\right]_{i}=\frac{1}{\left|K_{n}\right|} \int_{K_{n}} x_{i} d x}
\end{gathered}
$$

By Sc93, Theorem 1.8.16], the volume map is continuous in the Hausdorff metric. In other words, if $S_{n} \rightarrow S$, then $\left|S_{n}\right| \rightarrow|S|$. We now show that the center of mass map is also continuous with respect to the Hausdorff metric on the class of convex bodies. To prove this we need to demonstrate that $\int_{K_{n}} x_{i} d x \rightarrow \int_{K} x_{i} d x$. Since 
$\left\{K_{n}\right\}$ converges, there exists an $R>0$ such that $K, K_{n} \subset B_{R}(0)$ for all $n$. Then

$$
\begin{gathered}
\left|\int_{K_{n}} x_{i} d x-\int_{K} x_{i} d x\right| \leq \int_{K_{n} \cap K^{c}}\left|x_{i}\right| d x+\int_{K \cap K_{n}^{c}}\left|x_{i}\right| d x \\
\leq R\left(\left|K_{n} \cap K^{c}\right|+\left|K \cap K_{n}^{c}\right|\right) \rightarrow 0
\end{gathered}
$$

as $n \rightarrow \infty$, since by Lemma 5.5. $\chi_{K_{n}} \rightarrow \chi_{K}$ almost everywhere.

Now let $\epsilon>0$. Then there exists $N$ such that $n \geq N$ implies that $K \subset K_{n}+\epsilon B_{1}$, $K_{n} \subset K+\epsilon B_{1}$, and $\left|c\left(K_{n}\right)-c(K)\right| \leq \epsilon$. We need to show that there exists $N^{\prime}$ such that if $n \geq N^{\prime}$, the following two inclusions hold: $\frac{1}{2} K \subset \frac{1}{2} K_{n}+\epsilon B_{1}$ and $\frac{1}{2} K_{n} \subset \frac{1}{2} K+\epsilon B_{1}$.

Let $x \in \frac{1}{2} K$. Then $x=\frac{1}{2}(c(K)+y)$ for some $y \in K$. The first inclusion will be proved if, for all $n$ large enough, there is a point $z_{n} \in \frac{1}{2} K_{n}$ such that $\left|x-z_{n}\right| \leq \epsilon$. For each $n \geq N$, there is a point $\bar{z}_{n} \in K_{n}$ such that $\left|\bar{z}_{n}-y\right| \leq \epsilon$. Let $z_{n}=\frac{1}{2}\left(c\left(K_{n}\right)+\bar{z}_{n}\right) \in \frac{1}{2} K_{n}$. Then

$$
\left|x-z_{n}\right|=\left|\frac{1}{2}(c(K)+y)-\frac{1}{2}\left(c\left(K_{n}\right)+\bar{z}_{n}\right)\right| \leq \frac{1}{2}\left(\left|c(K)-c\left(K_{n}\right)\right|+\left|y-\bar{z}_{n}\right|\right) \leq \epsilon .
$$

The other inclusion is proved by contradiction. If the claim is not true, then there exist $\epsilon>0$ and a subsequence $K_{n_{j}}$ such that $\frac{1}{2} K_{n_{j}} \not \subset \frac{1}{2} K+\epsilon B_{1}(0)$. This means that we can find a sequence of points $\left\{x_{n_{j}}\right\}$ such that $x_{n_{j}} \in \frac{1}{2} K_{n_{j}}$ and $\operatorname{dist}\left(x_{n_{j}}, \frac{1}{2} K\right)>\epsilon$ for all points in the subsequence. Write $x_{n_{j}}=\frac{1}{2}\left(c\left(K_{n_{j}}\right)+\bar{x}_{n_{j}}\right)$ where $\bar{x}_{n_{j}} \in K_{n_{j}}$. By passing to another subsequence, we can assume that $\bar{x}_{n_{j}} \rightarrow \bar{x}$. Then by letting $j \rightarrow \infty$, we see that $x_{n_{j}}$ also approaches a limit, namely $\frac{1}{2}(c(K)+\bar{x})$. Theorem 1.8.7 (b) in [Sc93] states that if $K_{n} \rightarrow K$ and $\left\{x_{n_{j}}\right\}$ converges to $x$, where $x_{n_{j}} \in K_{n_{j}}$, then $x \in K$. This theorem implies that $\bar{x} \in K$, and therefore $\left\{x_{n_{j}}\right\}$ converges to a point in $\frac{1}{2} K$. However, this is impossible if $\operatorname{dist}\left(x_{n_{j}}, \frac{1}{2} K\right)>\epsilon$.

The next two results in this section concern the convergence of the normalized distances to the boundaries of convex sets converging in the Hausdorff sense, and the continuity of sections in the parameter $t$.

Lemma 5.7. Let $\left\{S_{j}\right\}$ be a sequence of convex bodies converging to the convex body $S$. Then for every $x \in S^{\circ}, \delta\left(x, S_{j}\right) \rightarrow \delta(x, S)$. In fact, the functions $f_{j}(x)=$ $\delta\left(x, S_{j}\right)$ converge uniformly to $f(x)=\delta(x, S)$ on compact subsets of $S$.

Proof. Let $x \in S^{\circ}$. Then $\operatorname{dist}(x, \partial S)=\rho>0$. Since $S_{j} \rightarrow S$, Lemma 5.5 implies that $x \in S_{j}$ for all $j$ sufficiently large (depending on $\rho$ ). Then for these $j, \delta\left(x, S_{j}\right)$ is defined.

Let $l$ be any line through $x$. Let $x_{1}$ and $x_{2}$ be the endpoints of the segment $l \cap \bar{S}$. Let $x_{1}^{j}$ and $x_{2}^{j}$ be the endpoints of the segment $l \cap \bar{S}_{j}$, with $x_{1}^{j}$ being in the same ray (emanating from $x$ ) as $x_{1}$. We make the claim that $x_{1}^{j} \rightarrow x_{1}$ and $x_{2}^{j} \rightarrow x_{2}$ "uniformly" in the sense that this convergence does not depend on $l$.

Then

$$
\frac{\left|x_{1}^{j}-x\right|}{\left|x_{2}^{j}-x\right|} \rightarrow \frac{\left|x_{1}-x\right|}{\left|x_{2}-x\right|},
$$

and this implies that $\delta\left(x, S_{j}\right) \rightarrow \delta(x, S)$.

We prove the claim by considering two cases. First, consider the case that $\left|x_{1}^{j}-x\right|>\left|x_{1}-x\right|$. Let $u$ be the unit vector from $x$ along $l$, pointing in the direction of $x_{1}$. Let $\Pi$ be a support plane to $S$ at $x_{1}$; let $v$ be its unit normal (away from $S$ ). Let $\Pi_{j}$ be the plane parallel to $\Pi$ that supports $S_{j}$ at some point. Let 
$R_{j}=\operatorname{dist}\left(\Pi, \Pi_{j}\right)$. Let $\theta$ be the angle between $u$ and $v$. Then $\left|x_{1}^{j}-x_{1}\right| \leq R_{j} \sec \theta$. To estimate $\sec \theta$, we construct a right triangle with one vertex at $x$ in the following way. The angle at $x$ is $\theta$, and the sides intersecting at $x$ are given by the rays emanating from $x$ with directions $u$ and $v$. The second vertex $A$ is the point in $\partial S$ where the ray starting at $x$ in the direction $v$ hits $\partial S$. The third vertex is the point $B$ lying in the ray from $x$ with direction $u$ that lies in a plane parallel to $\Pi$ through A. Then

$$
\cos \theta=\frac{|x-A|}{|x-B|} \geq \frac{\rho}{\operatorname{diam}(S)}>0 .
$$

Hence, there is a number $M$ for which $\sec \theta<M$. Therefore, $\left|x_{1}^{j}-x_{1}\right| \leq M R_{j}$. By Theorem 5.3, $R_{j} \leq d_{H}\left(S_{j}, S\right) \rightarrow 0$. This is because the number $R_{j}$ is $h\left(v, S_{j}\right)-$ $h(v, S)$, where $h(\cdot, \cdot)$ is the Minkowski support function. This shows that $x_{1}^{j} \rightarrow x_{1}$ at least for those $j$ such that $\left|x_{1}^{j}-x\right|>\left|x_{1}-x\right|$.

We now consider the other possibility, that $\left|x_{1}-x\right|>\left|x_{1}^{j}-x\right|$. As before, let $u$ be a unit vector from $x$ along $l$ pointing in the direction of $x_{1}$. Let $\Pi_{j}$ be a support plane to $S_{j}$ at $x_{1}^{j}$ and let $v_{j}$ be its unit normal (pointing away from $S_{j}$ ). Let $\Pi_{j}^{\prime}$ be parallel to $\Pi_{j}$ and support $S$ at some point. Denote by $R_{j}$ the distance between the parallel planes $\Pi_{j}$ and $\Pi_{j}^{\prime}$. Let $\theta_{j}$ be the angle between $u$ and $v_{j}$. Then $\left|x_{1}^{j}-x_{1}\right| \leq R_{j} \sec \theta_{j}$. For each $j$ we construct a right triangle with vertex $x$ and sides intersecting at $x$ given by the rays starting at $x$ with directions $u$ and $v_{j}$. The second vertex of the triangle, $A_{j}$, lies at the end of the side with direction $v_{j}$ and is in the boundary of $S_{j}$. The third vertex, $B_{j}$, is found by intersecting the side with direction $u$ with the plane parallel to $\Pi_{j}$ that passes through $A_{j}$. Then

$$
\cos \theta_{j}=\frac{\left|x-A_{j}\right|}{\left|x-B_{j}\right|} \geq \frac{\rho / 2}{\operatorname{diam}(S)}>0 .
$$

Then, as in the first case, $x_{1}^{j} \rightarrow x_{1}$ for those $j$ for which $\left|x_{1}-x\right|>\left|x_{1}^{j}-x\right|$.

For both of the cases considered, the same arguments show the corresponding result for $x_{2}$. Combining the two cases, we get that $x_{1}^{j} \rightarrow x_{1}$ and $x_{2}^{j} \rightarrow x_{2}$. Notice that the convergence does not depend on the particular line $l$. This allows us to conclude that $\delta\left(x, S_{j}\right) \rightarrow \delta(x, S)$ pointwise for every $x \in S^{\circ}$. The claim about uniform convergence on compact subsets follows, since the only property of the point $x$ needed in the above argument was its distance from the boundary of $S$.

Lemma 5.8. Let $u$ be a convex function defined on a domain $\Omega$. Let $S=S\left(x_{0}, p, t\right)$ be a section of $u$, with $S \Subset \Omega$. Let $\rho>0$. Denote by $S^{\rho}$ the section $S\left(x_{0}, p, t-\rho\right)$, and by $S_{\rho}$ the section $S\left(x_{0}, p, t+\rho\right)$. Then

$$
\lim _{\rho \rightarrow 0} S^{\rho}=\lim _{\rho \rightarrow 0} S_{\rho}=S
$$

where the limits are in the Hausdorff metric.

Proof. Let $l(x)=u\left(x_{0}\right)+p \cdot\left(x-x_{0}\right)+t$ be the affine function defining $S$, i.e., $S=\{x \in \Omega: u(x)<l(x)\}$. First we show that $\lim _{\rho \rightarrow 0} S_{\rho}=S$. For every $\rho$ we have $S \subset S_{\rho}$; so we only need to prove that, for every $\rho_{0}>0$,

$$
S_{\rho} \subset S+\rho_{0} B_{1}(0)
$$

for all $\rho$ sufficiently small. If this is not true, then there exists $\rho_{0}>0$ such that for each $n \in \mathbb{N}$ there exist $\epsilon_{n}<\frac{1}{n}$ and a point $x_{n} \in \partial S_{\epsilon_{n}} \cap\left(S+\rho_{0} B_{1}(0)\right)^{c}$. This implies 
that $\operatorname{dist}\left(x_{n}, \partial S\right)>\rho_{0}$. But this leads to a contradiction. Choose a subsequence $x_{n_{j}} \rightarrow \bar{x}$. Then

$$
\begin{array}{lll}
u\left(x_{n_{j}}\right) & = & u\left(x_{0}\right)+p \cdot\left(x_{n_{j}}-x_{0}\right)+t+\epsilon_{n_{j}} \\
\downarrow & & \\
u(\bar{x}) & = & u\left(x_{0}\right)+p \cdot\left(\bar{x}-x_{0}\right)+t .
\end{array}
$$

This means that $\bar{x} \in \partial S$, but $\operatorname{dist}(\bar{x}, \partial S)>\rho_{0}$. This is a contradiction.

Now we prove that $S^{\rho} \rightarrow S$ as $\rho \rightarrow 0$. Since $S^{\rho} \subset S$ for all $\rho$, we only need to show that, for all $\rho_{0}>0$,

$$
S \subset S^{\rho}+\rho_{0} B_{1}(0)
$$

for all $\rho$ sufficiently small. Again, the proof of this inclusion is by contradiction. If this does not hold, there exists a $\rho_{0}>0$ such that for all $n$, there exists $\epsilon_{n}<\frac{1}{n}$ such that $S \not \subset S^{\epsilon_{n}}+\rho_{0} B_{1}(0)$. So there is a point $x_{n} \in S$ such that $x_{n} \notin S^{\epsilon_{n}}+\rho_{0} B_{1}(0)$, meaning that $\operatorname{dist}\left(x_{n}, \overline{S^{\epsilon_{n}}}\right)>\rho_{0}$. Then we can choose a subsequence $x_{n_{j}} \rightarrow \bar{x} \in \bar{S}$. Each $x_{n_{j}} \notin S^{\epsilon_{n_{j}}}$, and so

$$
l\left(x_{n_{j}}\right)-\epsilon_{n_{j}} \leq u\left(x_{n_{j}}\right)<l\left(x_{n_{j}}\right) .
$$

Let $j \rightarrow \infty$ to conclude that $l(\bar{x}) \leq u(\bar{x}) \leq l(\bar{x})$. This means that $\bar{x} \in \partial S$. Since $\left|\operatorname{dist}\left(\bar{x}, \overline{S^{\epsilon_{n_{j}}}}\right)-\operatorname{dist}\left(x_{n_{j}}, \overline{S^{\epsilon_{n_{j}}}}\right)\right| \leq\left|\bar{x}-x_{n_{j}}\right|$, it follows that $\operatorname{dist}\left(\bar{x}, \overline{S^{\epsilon_{n_{j}}}}\right) \geq \frac{\rho_{0}}{2}$ for all $j$ large enough. Therefore, $B_{\frac{\rho_{0}}{2}}(\bar{x}) \cap S^{\epsilon_{n_{j}}}=\emptyset$ for large $j$. However, since $\bar{x} \in \partial S$, there is a point $z \in B_{\frac{\rho_{0}}{2}}(\bar{x})$ such that $z \in S$. This means that $u(z)<l(z)$. So $u(z)<$ $l(z)-\epsilon_{n_{j}}$ for $j$ large enough, implying that $z \in S^{\epsilon_{n_{j}}}$. This is a contradiction.

Lemma 5.9. Let $\Omega \subset \mathbb{R}^{n}$ be open, bounded and convex, and let $u \in C(\bar{\Omega})$ be convex with $\left.u\right|_{\partial \Omega}=0$. Suppose $M u \in D_{\epsilon}$ for some $\epsilon \in(0,1]$. Then

$$
\int_{\Omega} \delta(x, \Omega)^{1-\epsilon} d M u \leq C M u\left(\frac{1}{2} \Omega\right),
$$

where $C$ is the $D_{\epsilon}$ constant.

Proof. If $u$ is identically zero, the claim is trivial. Notice that, from Definition 2.6, at this point we only know that (5.1) holds for all sections $S$ of $u$ compactly contained in $\Omega$. To prove (5.1), let $D_{k}=\{x \in \Omega: u(x)<-1 / k\}$ and notice that since $u=0$ on $\partial \Omega$ and $u$ is nontrivial, the set $D_{k}$ is a section of $u$ compactly contained in $\Omega$, and so (5.1) holds for $D_{k}$ replacing $\Omega$. Following the argument used in the proof of the second part of Lemma 5.8, we have that $D_{k} \rightarrow \Omega$ in the Hausdorff metric as $k \rightarrow \infty$. Hence by Lemmas 5.5 and 5.7 we get that $\chi_{D_{k}}(x) \delta\left(x, D_{k}\right) \rightarrow \chi_{\Omega}(x) \delta(x, \Omega)$ for a.e. $x \in \Omega$ as $k \rightarrow \infty$, and so, by Fatou's lemma,

$$
\int_{\Omega} \delta(x, \Omega)^{1-\epsilon} d M u \leq \liminf \int \chi_{D_{k}}(x) \delta\left(x, D_{k}\right)^{1-\epsilon} d M u .
$$

Since $D_{k} \rightarrow \Omega, \frac{1}{2} D_{k} \rightarrow \frac{1}{2} \Omega$ by Lemma [5.6] From Lemma [5.5, we get that $\chi_{\frac{1}{2} D_{k}} \rightarrow \chi_{\frac{1}{2} \Omega}$ pointwise a.e. Also, since $\frac{1}{2} D_{k} \rightarrow \frac{1}{2} \Omega$, we have $\frac{1}{2} D_{k} \subset \frac{3}{4} \Omega$ for $k$ sufficiently large. Therefore, $\chi_{\frac{1}{2} D_{k}}(x) \leq \chi_{\frac{3}{4} \Omega}(x) \in L^{1}(\Omega, M u)$. So by the dominated convergence theorem, $M u\left(\frac{1}{2} D_{k}\right) \rightarrow M u\left(\frac{1}{2} \Omega\right)$. This proves the lemma. 


\section{Selection Lemma and a Uniform Height for Sections}

In this section we prove two main results that are needed for the regularity theory appearing in the following section. The first of these concerns the selection of a convergent subsequence from a sequence of solutions to Dirichlet problems in normalized domains under the hypotheses of the $D_{\epsilon}$ condition and uniform local boundedness of the corresponding Monge-Ampère measures. The first application of this selection result is to establish that if $u$ is zero on the boundary of a normalized domain $\Omega$ and $M u \in D_{\epsilon}$ and $M u$ does not grow too quickly near the boundary, then any section of $u$ up to a certain height (depending only on the structure and the distance the base point of the section is away from $\partial \Omega$ ) will be compactly contained inside $\Omega$. These results are the analogues of Lemma 5.3.1 and Theorem 5.3.3 in G01.

Lemma 6.1 (Selection Lemma). Let $\left\{\Omega_{j}\right\}_{1}^{\infty}$ be a sequence of normalized convex domains, and let $u_{j} \in C\left(\bar{\Omega}_{j}\right)$ be convex, $\left.u_{j}\right|_{\partial \Omega_{j}}=0$, with $M u_{j} \in D_{\epsilon}(C)$ for all $j$. Assume also that $M u_{j}$ is absolutely continuous with respect to Lebesgue measure for each $j$ with density $d_{j}(x)$ that is locally uniformly bounded, i.e., for each compact $K \subset \Omega_{j}$ there exists a constant $C_{K}$ such that $d_{j}(x) \leq C_{K}$ for a.e. $x \in K$ and for all $j$. Then if $0<\lambda \leq\left|\inf _{\Omega_{j}} u_{j}\right|$ for all $j$, there exist:

(a) a normalized convex domain $\Omega_{0}$,

(b) $u_{0} \in C\left(\bar{\Omega}_{0}\right)$, convex, with $M u_{0} \in D_{\epsilon}(C), M u_{0}$ absolutely continuous with respect to Lebesgue measure with density $d_{0}(x)$ locally bounded in $\Omega_{0},\left.u_{0}\right|_{\partial \Omega_{0}}=$ 0 , and $\lambda \leq\left|\inf u_{0}\right|$; and a subsequence of the $u_{j}$ that converges uniformly on compact subsets to $u_{0}$.

If, in addition, for each $j$, there exists $x_{j} \in \Omega_{j}$ with $\operatorname{dist}\left(x_{j}, \partial \Omega_{j}\right) \geq \epsilon^{\prime}$, and $l_{j}(x)$ a support plane to $u_{j}$ at $x_{j}$ such that

$$
S_{j}=\left\{x \in \Omega_{j}: u_{j}(x)<l_{j}(x)+\frac{1}{j}\right\} \not \subset\left\{x \in \Omega_{j}: u_{j}(x)<-\bar{C} \epsilon^{\prime}\right\},
$$

then there exist:

(c) a point $x_{0} \in \Omega_{0}$ such that $\operatorname{dist}\left(x_{0}, \partial \Omega_{0}\right) \geq \epsilon^{\prime}$, and

(d) a support plane $l_{0}$ to $u_{0}$ at $x_{0}$ such that

$$
S_{0}=\left\{x \in \Omega_{0}: u_{0}(x)=l_{0}(x)\right\} \not \subset\left\{x \in \Omega_{0}: u_{0}(x)<-\bar{C} \epsilon^{\prime}\right\}=T_{0} .
$$

Before starting the proof of this lemma, we make a few remarks.

Remark 6.2. The boundedness condition on the densities $d_{j}$ is necessary (and restricting just the $D_{\epsilon}$ constant is not enough) to guarantee the existence of a uniformly convergent subsequence, as the following example demonstrates. For each positive integer $N$ we can uniquely solve the problem (provided $\Omega$ is strictly convex)

$$
\begin{cases}\operatorname{det} D^{2} u & =N d x \\ \left.u\right|_{\partial \Omega} & =0\end{cases}
$$

The density of the measure $M u_{N}$ corresponding to the solution $u_{N}$ is the constant function $N$; so the boundedness condition is not met. Then for any section $S_{N}$ of $u_{N}, M u_{N}\left(S_{N}\right)=N\left|S_{N}\right|$ and $M u_{N}\left(\frac{1}{2} S_{N}\right)=N\left|\frac{1}{2} S_{N}\right|=\frac{1}{2^{n}} N\left|S_{N}\right|$. Therefore, $M u_{N}$ is doubling on the sections of $u_{N}$ for all $N$ with the same doubling constant. If $\Omega$ is normalized, Proposition 2.10 and Lemma 5.9 tell us that $\left|\min _{\Omega} u_{N}\right|^{n} \approx N$. From this, one sees that the sequence $\left\{\min _{\Omega} u_{N}\right\}$ is unbounded, and therefore, $\left\{u_{N}\right\}$ cannot have a uniformly convergent subsequence. 
Remark 6.3. If a measure $\mu \in D_{\epsilon}$, it is possible for $\mu$ to have singular part with respect to Lebesgue measure. Indeed, there exists a measure that is doubling on intervals in $\mathbb{R}$ and that is totally singular with respect to $d x$. See [St93, p. 40] for details.

Proof of Lemma 6.1. The domain $\Omega_{0}$ can be produced by the Blaschke Selection Theorem 5.4. The sequence $\left\{\overline{\Omega_{j}}\right\}$ is a bounded sequence of convex bodies. So it has a subsequence (which we also denote with $\left\{\overline{\Omega_{j}}\right\}$ ) converging in the Hausdorff metric to a convex body, say $\overline{\Omega_{0}}$; we can take $\Omega_{0}$ to be the interior of this compact set. Then, given $\rho>0$, for all $j$ sufficiently large we have

$$
\Omega_{j} \subset \Omega_{0}+\rho B_{1}(0) \quad \text { and } \quad \Omega_{0} \subset \Omega_{j}+\rho B_{1}(0) .
$$

Since each $\Omega_{j}$ is normalized, these inclusions imply that $\Omega_{0}$ is as well. This demonstrates $(a)$.

The proof of the rest of the theorem will be done in stages.

Step 1. The first step in proving $(b)$ is to show that for every compact $K \Subset \Omega_{0}$, there are positive constants $j_{0}(K)$ and $c(K)$ such that

$$
K \subset\left\{x \in \Omega_{j}: \operatorname{dist}\left(x, \partial \Omega_{j}\right)>c(K)\right\}
$$

for all $j \geq j_{0}(K)$.

Let $\operatorname{dist}\left(K, \partial \Omega_{0}\right)=\rho>0$, and let $x \in K$. Translate the coordinates so that $x$ is now 0 . Let $\tilde{\Omega}_{j}=\Omega_{j}-x$, and $\tilde{\Omega}_{0}=\Omega_{0}-x$. Since $\Omega_{j} \rightarrow \Omega_{0}$, there exists $J$ such that if $j \geq J$, then $d_{H}\left(\Omega_{j}, \Omega_{0}\right)<\frac{\rho}{2}$. Since $d_{H}$ is invariant under translations, $d_{H}\left(\Omega_{j}, \Omega_{0}\right)=$ $d_{H}\left(\tilde{\Omega}_{j}, \tilde{\Omega}_{0}\right)$. By Theorem[5.3, for any $u \in S^{n-1}, d_{H}\left(\tilde{\Omega}_{j}, \tilde{\Omega}_{0}\right) \geq\left|h\left(\tilde{\Omega}_{j}, u\right)-h\left(\tilde{\Omega}_{0}, u\right)\right|$. We have that $h\left(\tilde{\Omega}_{0}, u\right) \geq \rho$ for all unit vectors $u$, since $B_{\rho}(0) \subset \tilde{\Omega}_{0}$. Therefore,

$$
\frac{\rho}{2} \geq\left|h\left(\tilde{\Omega}_{j}, u\right)-h\left(\tilde{\Omega}_{0}, u\right)\right| \geq \rho-\left|h\left(\tilde{\Omega}_{j}, u\right)\right| .
$$

This implies that $h\left(\tilde{\Omega}_{j}, u\right) \geq \frac{\rho}{2}$. Therefore, $B_{\frac{\rho}{2}}(0) \subset \tilde{\Omega}_{j}$; translating back we obtain that $B_{\frac{\rho}{2}}(x) \subset \Omega_{j}$ for all $j \geq J$. Since $x \in K$ was arbitrary, we have that $\operatorname{dist}\left(K, \partial \Omega_{j}\right) \geq \frac{\rho}{2}$ for $j$ sufficiently large.

Step 2. In this step, we prove that $\left|\min _{\Omega_{j}} u_{j}\right|$ is bounded above.

Suppose that the sequence of minima is not bounded. Then there is a subsequence such that $\min _{\Omega_{j}} u_{j}<-j$. Then, by Theorem 2.8 and Lemma 5.9 .

$$
j^{n} \leq C \int_{\Omega_{j}} \delta\left(x, \Omega_{j}\right)^{1-\epsilon} d M u_{j} \leq C M u_{j}\left(\frac{1}{2} \Omega_{j}\right) .
$$

This implies that $M u_{j}\left(\frac{1}{2} \Omega_{j}\right) \rightarrow \infty$. But we have that $\Omega_{j} \rightarrow \Omega_{0}$. So by Lemma 5.6 $\frac{1}{2} \Omega_{j} \rightarrow \frac{1}{2} \Omega_{0}$, so that for large $j, \frac{1}{2} \Omega_{j} \subset \frac{3}{4} \Omega_{0}$. Note also that for large $j, \frac{3}{4} \Omega_{0} \subset \Omega_{j}$. Hence

$$
M u_{j}\left(\frac{1}{2} \Omega_{j}\right) \leq M u_{j}\left(\frac{3}{4} \Omega_{0}\right)=\int_{\frac{3}{4} \Omega_{0}} d_{j}(x) d x,
$$

but these integrals are bounded above by the uniform local boundedness. Therefore there exists a positive $\Lambda$ such that $\left|\min _{\Omega_{j}} u_{j}\right| \leq \Lambda$ for all $j$.

Step 3. Now we show that for every compact $K \Subset \Omega_{0}$, there is a constant $C(K)$ such that for every $x \in K$ and every $p \in \partial u_{j}(x)$,

$$
\left|u_{j}(x)\right|+|p| \leq C(K)
$$


By the last step, the $\left\{u_{j}\right\}$ are uniformly bounded. Also by virtue of the first step, $\rho>0$ can be chosen so that

$$
K_{\rho}=\{x: \operatorname{dist}(x, K) \leq \rho\} \Subset \Omega_{j}
$$

for $j \geq j_{0}(K)$. Let $0 \neq p \in \partial u_{j}(K)$ for any $j \geq j_{0}(K)$, say $p \in \partial u_{j}(\bar{x})$, where $\bar{x} \in K$. Then

$$
u_{j}(x) \geq u_{j}(\bar{x})+p \cdot(x-\bar{x})
$$

for all $x \in \Omega_{j}$. In particular, this is true for $x=\bar{x}+\rho \omega$, where $|\omega|=1$. Then

$$
u_{j}(\bar{x}+\rho \omega) \geq u_{j}(\bar{x})+\rho|p|, \quad \text { implying } \max _{K_{\rho}} u_{j} \geq \min _{K} u_{j}+\rho|p|,
$$

and therefore,

$$
|p| \leq \frac{\max _{K_{\rho}} u_{j}-\min _{K} u_{j}}{\rho}<\infty,
$$

since by the previous step the $u_{j}$ are uniformly bounded.

Step 4. We now produce the function $u_{0}$. To begin, we demonstrate that given $K \Subset \Omega_{0}$, the $\left\{u_{j}\right\}$ are uniformly Lipschitz on $K$, for $j$ large enough, i.e., we show that $\left|u_{j}(x)-u_{j}(z)\right| \leq C(K)|x-z|$ for all $x, z \in K$ and for all $j \geq j_{0}(K)$. The proof of this claim is as in the proof of [G01, Lemma 1.1.6]. Let $x \in K$ and $p \in \partial u_{j}(x)$. Then by Step $3,|p| \leq C(K)$. For any $z \in K$,

$$
u_{j}(z) \geq u_{j}(x)+p \cdot(z-x) \quad \text { or } \quad u_{j}(z)-u_{j}(x) \geq-|p||z-x| \geq-C(K)|z-x| .
$$

We can reverse the roles of $x$ and $z$ to conclude that $\left|u_{j}(x)-u_{j}(z)\right| \leq C(K)|x-z|$ for all $j \geq j_{0}(K)$. In particular, the $\left\{u_{j}\right\}$ are equicontinuous on $K$.

Therefore, by Arzelà-Ascoli, there exists a uniformly convergent subsequence in $K$. Write $\Omega_{0}=K_{1} \cup K_{2} \cup K_{3} \cup \cdots$, with $K_{1} \Subset K_{2} \Subset \cdots$. By a diagonal process, we can extract a subsequence of the $u_{j}$ that converges uniformly on compact subsets of $\Omega_{0}$. Define $u_{0}(x)$ to be the limit of this subsequence.

Because $u_{0}$ is the limit of convex functions, it is convex. If $x \in \Omega_{0}$, then $x \in \Omega_{j}$ for $j$ large. Then, since $\left.u_{j}\right|_{\partial \Omega_{j}}=0, u_{j}(x) \leq 0$. By letting $j \rightarrow \infty$, we get that $u_{0}(x) \leq 0$. Also, since $\lambda \leq\left|\min u_{j}\right|$ for every $j$, we have that $\lambda \leq\left|\min u_{0}\right|$.

Step 5. The next step is to show that $u_{0} \in C\left(\bar{\Omega}_{0}\right)$ and $\left.u_{0}\right|_{\partial \Omega_{0}}=0$. To this end, we first show that for every $\eta>0$, there is a number $j_{0}(\eta)$ such that

$$
\left\{x \in \Omega_{j}: \operatorname{dist}\left(x, \partial \Omega_{j}\right) \geq \eta\right\} \subset\left\{x \in \Omega_{0}: \operatorname{dist}\left(x, \partial \Omega_{0}\right) \geq \frac{\eta}{2}\right\},
$$

for all $j \geq j_{0}(\eta)$. This can be shown by an argument similar to that in Step 1 . For $j \geq J_{1}$, we have that $d_{H}\left(\Omega_{j}, \Omega\right)<\frac{\eta}{2}$. Let $j \geq J_{1}$ and let $x \in \Omega_{j}$ satisfy $\operatorname{dist}\left(x, \partial \Omega_{j}\right) \geq \eta$. Define $\tilde{\Omega}_{j}=\Omega_{j}-x$ and $\tilde{\Omega}_{0}=\Omega_{0}-x$. This change of coordinates takes $x$ to the origin, and, as before, we have that $d_{H}\left(\tilde{\Omega}_{j}, \tilde{\Omega}_{0}\right)=d_{H}\left(\Omega_{j}, \Omega_{0}\right)$. Then for any $u \in S^{n-1}$ (by Theorem 5.3)

$$
\frac{\eta}{2}>d_{H}\left(\tilde{\Omega}_{j}, \tilde{\Omega}_{0}\right) \geq\left|h\left(\tilde{\Omega}_{j}, u\right)-h\left(\tilde{\Omega}_{0}, u\right)\right| .
$$

Since $B_{\eta}(0) \subset \tilde{\Omega}_{j}, h\left(\tilde{\Omega}_{j}, u\right) \geq \eta$. Hence, $h\left(\tilde{\Omega}_{0}, u\right) \geq \frac{\eta}{2}$; so $B_{\frac{\eta}{2}}(0) \subset \tilde{\Omega}_{0}$. This last inclusion is equivalent to $B_{\frac{\eta}{2}}(x) \subset \Omega_{0}$, and (6.3) holds.

We now show that

$$
\left\{x \in \Omega_{0}: u_{0}(x)<-\rho\right\} \Subset \Omega_{0}
$$

for each $\rho>0$. 
Using Theorem [2.8, Proposition 2.10, and Lemma 5.9] we get that

$$
\left|u_{j}(x)\right|^{n} \leq C \delta\left(x, \Omega_{j}\right)^{\epsilon} \int_{\Omega_{j}} \delta\left(y, \Omega_{j}\right)^{1-\epsilon} d M u_{j} \leq C \Lambda^{n} \delta\left(x, \Omega_{j}\right)^{\epsilon} \leq \tilde{C} \operatorname{dist}\left(x, \Omega_{j}\right)^{\epsilon} .
$$

This implies that $\left|u_{j}(x)\right|^{\frac{n}{\epsilon}} \leq \tilde{C} \operatorname{dist}\left(x, \Omega_{j}\right)$. So if $u_{j}(x)<\frac{-\rho}{2}$, then

$$
\left(\frac{\rho}{2}\right)^{\frac{n}{\epsilon}} \leq C \operatorname{dist}\left(x, \partial \Omega_{j}\right) .
$$

In other words, the following inclusion holds:

$$
\left\{x \in \Omega_{j}: u_{j}(x)<\frac{-\rho}{2}\right\} \subset\left\{x \in \Omega_{j}: \operatorname{dist}\left(x, \partial \Omega_{j}\right) \geq C \rho^{\frac{n}{\epsilon}}\right\} .
$$

Then by (6.3), for $j$ large enough (depending on $\rho$ ),

$$
\left\{x \in \Omega_{j}: \operatorname{dist}\left(x, \partial \Omega_{j}\right) \geq C \rho^{\frac{n}{\epsilon}}\right\} \subset\left\{x \in \Omega_{0}: \operatorname{dist}\left(x, \partial \Omega_{0}\right) \geq \frac{C}{2} \rho^{\frac{n}{\epsilon}}\right\} \equiv K(\rho) .
$$

Therefore,

$$
\bigcup_{j \geq j_{0}(\rho)}\left\{x \in \Omega_{j}: u_{j}(x)<\frac{-\rho}{2}\right\} \subset K(\rho) .
$$

This implies that $\left\{x \in \Omega_{0}: u_{0}(x)<-\rho\right\} \subset K(\rho)$. This is because if $x \in \Omega_{0}$, then $x \in \Omega_{j}$ for all $j$ large enough (by Lemma 5.5), and if $u_{0}(x)<-\rho$, then $u_{j}(x)<-\frac{\rho}{2}$ for all large $j$. This proves that

$$
\lim _{x \rightarrow \partial \Omega_{0}} u_{0}(x)=0 .
$$

Step 6. The longest and most delicate part of the proof is to show that $M u_{0} \in$ $D_{\epsilon}(C)$.

Since $u_{j} \rightarrow u_{0}$ uniformly on compact subsets, the measures $M u_{j}$ converge to $M u_{0}$ weakly by Lemma 2.1 .

We begin by showing that

(i) $M u_{0}$ is absolutely continuous with respect to Lebesgue measure, and its density is locally bounded;

(ii) for each measurable set $E$ with $\bar{E} \subset \Omega_{0}$, we have

$$
M u_{j}(E) \rightarrow M u_{0}(E) .
$$

Let $K \subset \Omega_{0}$ be a compact set, and let $E \subset K$ be a measurable set with $|E|=0$. Consider $G_{0}$ open such that $K \subset G_{0}$ and $\bar{G}_{0} \subset \Omega_{0}$. Given $\epsilon>0$, there exists an open set $G \subset \bar{G}_{0}$ such that $E \subset G$ and $|G|<\epsilon$. Let $F$ be an arbitrary closed subset of $E$, and let $\phi \in C_{0}\left(\Omega_{0}\right)$ be such that $\phi=1$ in $F, \phi=0$ on $G^{c}$, and $0 \leq \phi \leq 1$. We have $d_{j}(x) \leq C_{\bar{G}_{0}}$ for all $j$ and for a.e. $x \in \bar{G}_{0}$; so

$$
\int \phi d M u_{j} \leq M u_{j}(G) \leq C_{\bar{G}_{0}}|G| \leq C_{\bar{G}_{0}} \epsilon .
$$

Letting $j \rightarrow \infty$, we get

$$
M u_{0}(F) \leq \int \phi d M u_{0}<C_{\bar{G}_{0}} \epsilon
$$

and consequently $M u_{0}(F)=0$ for each closed set $F \subset E$. Since the measure $M u_{0}$ is regular, we get $M u_{0}(E)=0$, and the absolute continuity of $M u_{0}$ follows. To show that $d_{0}$ is locally bounded, let $K \subset \Omega_{0}$ be a compact set and let $B_{r}(x)$ be a ball with $x \in K$ and $r<\operatorname{dist}\left(K, \partial \Omega_{0}\right) / 2$. Let $K^{\prime}$ be the compact set containing $K$ and all these balls $B_{r}(x)$ with $x \in K$. We have $K^{\prime} \subset \Omega_{0}$. Let $\epsilon>0$, and 
consider $\phi \in C_{0}\left(\Omega_{0}\right)$ with $\phi=1$ on $B_{r-\epsilon}(x)$ and $\phi=0$ on $B_{r}(x)^{c}, 0 \leq \phi \leq 1$. From the local uniform boundedness of $d_{j}$ we have $\int_{B_{r}(x)} \phi d_{j} d x \leq C_{K^{\prime}}\left|B_{r}(x)\right|$, and then, from the weak convergence, $\int_{B_{r}(x)} \phi d_{0} d x \leq C_{K^{\prime}}\left|B_{r}(x)\right|$. Consequently $\int_{B_{r-\epsilon}(x)} d_{0} d x \leq C_{K^{\prime}}\left|B_{r}(x)\right|$. Letting first $\epsilon \rightarrow 0$ and then $r \rightarrow 0$, we get by differentiation that $d_{0}(x) \leq C_{K^{\prime}}$ for a.e. $x \in K$. We now prove (ii). Let $\epsilon>0$, $F \subset \Omega_{0}$ a closed set and let $G$ be open such that $F \subset G, G \subset K^{\prime}$ with $K^{\prime}$ compact, $K^{\prime} \subset \Omega_{0}$, and $|G \backslash F| \leq \epsilon$. Let $f \in C_{0}\left(\Omega_{0}\right)$ be such that $f=1$ on $F$ and $f=0$ on $G^{c}, 0 \leq f \leq 1$. We write

$$
\int_{G} f(x) d_{j}(x) d x=\int_{F} d_{j}(x) d x+\int_{G \backslash F} f(x) d_{j}(x) d x
$$

and

$$
\int_{G} f(x) d_{0}(x) d x=\int_{F} d_{0}(x) d x+\int_{G \backslash F} f(x) d_{0}(x) d x .
$$

Using the weak convergence of $M u_{j} \rightarrow M u_{0}$ and the locally uniform boundedness of $d_{j}$ and $d_{0}$, subtracting the previous identities we get that $M u_{j}(F) \rightarrow M u_{0}(F)$ as $j \rightarrow \infty$ for each closed set $F \subset \Omega_{0}$. If $E$ is measurable with $\bar{E} \subset \Omega$, then we pick $F \subset E$ closed with $|E \backslash F| \leq \epsilon$, and we obtain (6.4) as desired.

To establish that $M u_{0} \in D_{\epsilon}(C)$, the idea is to approximate $S$ by $\left\{S_{j}\right\}$, a sequence of sections of $u_{j}$, with the property that $S_{j} \rightarrow S$ in the Hausdorff metric. Once the possibility of this approximation is demonstrated, we will show that this implies that

$$
M u_{j}\left(\frac{1}{2} S_{j}\right) \rightarrow M u_{0}\left(\frac{1}{2} S\right)
$$

and

$$
\int_{S_{j}} \delta\left(x, S_{j}\right)^{1-\epsilon} d M u_{j} \rightarrow \int_{S} \delta(x, S)^{1-\epsilon} d M u_{0} .
$$

This will establish the claim, since for all $j$ we have $M u_{j} \in D_{\epsilon}(C)$.

Let $S$ be a section of $u_{0}, S=S\left(x_{0}, p, t\right), S \Subset \Omega_{0}$. Let $l(x)=u_{0}\left(x_{0}\right)+p \cdot\left(x-x_{0}\right)+t$. Since $l(x)<0$ for all $x \in \Omega_{0}$ and $\Omega_{j} \rightarrow \Omega_{0}$, it follows that $l(x)<0$ for all $x \in \Omega_{j}$, for $j \geq J_{0}$. Then $l(x)$ determines a section $S_{j}$ of $u_{j}$ in $\Omega_{j}$ at some point (slide $l(x)$ down until it touches the graph of $u_{j}$ at one point, which will be the desired base point), with some parameter (equal to the distance that $l(x)$ must be lowered). In other words, $S_{j}=\left\{x \in \Omega_{j}: u_{j}(x)<l(x)\right\}$. By the uniform convergence of the $u_{j}$ to $u_{0}$, for any $\rho>0$, there exists $J_{1}$ such that for all $j \geq J_{1}$,

$$
u_{0}(x)-\rho \leq u_{j}(x) \leq u_{0}(x)+\rho,
$$

for every $x \in U$, where $U$ is an open set satisfying $\bar{S} \subset \bar{U} \subset \Omega_{0}$. Take $\rho$ small enough that $S_{\rho}$ is compactly contained in $U$, and $t-\rho>0$. Then we have

$$
S^{\rho} \subset S_{j} \subset S_{\rho}, \quad j \geq \max \left(J_{0}, J_{1}\right) .
$$

Now by Lemma 5.8, we have that $\lim _{\rho \rightarrow 0} S^{\rho}=\lim _{\rho \rightarrow 0} S_{\rho}=S$, implying that $\lim _{j \rightarrow \infty} S_{j}=S$.

The next step is to prove (6.5). Since $S_{j} \rightarrow S$ in the Hausdorff metric, $\frac{1}{2} S_{j} \rightarrow \frac{1}{2} S$ by Lemma 5.6] Let $\rho>0$. We need to show that $\left|M u_{j}\left(\frac{1}{2} S_{j}\right)-M u_{0}\left(\frac{1}{2} S\right)\right|<\rho$ for all $j \geq J(\rho)$. 
Let $f \in C_{0}(S), 0 \leq f \leq 1, f \equiv 1$ on $\frac{1}{2} S$, be such that

$$
\left|\int_{S} f(x) d M u_{0}-M u_{0}\left(\frac{1}{2} S\right)\right|<\rho .
$$

Then by the weak convergence, there exists $J_{1}=J_{1}(\rho)$ such that if $j \geq J_{1}$, then $\left|\int_{S} f(x) d M u_{j}-\int_{S} f(x) d M u_{0}\right|<\rho$. Therefore,

$$
\begin{gathered}
\left|\int_{S} f(x) d M u_{j}-M u_{0}\left(\frac{1}{2} S\right)\right| \\
\leq\left|\int_{S} f(x) d M u_{j}-\int_{S} f(x) d M u_{0}\right|+\left|\int_{S} f(x) d M u_{0}-M u_{0}\left(\frac{1}{2} S\right)\right|<2 \rho,
\end{gathered}
$$

and the claim will follow if $\left|M u_{j}\left(\frac{1}{2} S_{j}\right)-\int_{S} f(x) d M u_{j}\right|<\rho$ for all $j$ sufficiently large. We estimate this quantity in the following way:

$$
\begin{aligned}
\mid M u_{j}\left(\frac{1}{2} S_{j}\right)- & \int_{S} f(x) d M u_{j} \mid \\
& \leq\left|M u_{j}\left(\frac{1}{2} S_{j}\right)-M u_{j}\left(\frac{1}{2} S\right)\right|+\left|M u_{j}\left(\frac{1}{2} S\right)-\int_{S} f(x) d M u_{j}\right|=I+I I .
\end{aligned}
$$

We begin by examining $I$ :

$$
I \leq \int\left|\chi_{\frac{1}{2} S_{j}}(x)-\chi_{\frac{1}{2} S}(x)\right| d M u_{j} \equiv \int f_{j} d M u_{j}
$$

We want to show that $\lim _{j \rightarrow \infty} \int f_{j} d M u_{j}=0$. We have that $f_{j} \rightarrow 0$ pointwise a.e. by Lemma 5.5 (with respect to $M u_{0}$; here is where the absolute continuity is needed) and $\left|f_{j}\right| \leq 1$ for all $j$. Since $\frac{1}{2} S_{j} \rightarrow \frac{1}{2} S$ and $\overline{\frac{1}{2} S} \subset \Omega_{0}$, there exists a set $A$ such that $\frac{1}{2} S_{j}, \frac{1}{2} S \subset \bar{A} \subset \Omega_{0}$, for all $j \geq J_{2}$. By Egorov's theorem, there is a set $E \subset A$, with $M u_{0}(E)<\rho$, such that $f_{j} \rightarrow 0$ uniformly on $A \backslash E$. Since $f_{j}$ can take on the values 0 and 1 only, this means that $f_{j}(x)=0$ for all $j \geq J_{3}$ and all $x \in A \backslash E$. Then

$$
\int f_{j} d M u_{j}=\int_{A} f_{j} d M u_{j}=\int_{E} f_{j} d M u_{j}+\int_{A \backslash E} f_{j} d M u_{j} \leq M u_{j}(E) .
$$

By (6.4) we have $\left|M u_{j}(E)-M u_{0}(E)\right| \leq \rho$ for $j \geq J_{4}$, implying that $M u_{j}(E) \leq 2 \rho$. Therefore, $I \leq 2 \rho$ for $j \geq \max \left(J_{1}, J_{2}, J_{3}, J_{4}\right)$.

We now consider the second integral:

$$
I I=\int_{S} f(x) d M u_{j}-M u_{j}\left(\frac{1}{2} S\right)=\int_{S \backslash\left(\frac{1}{2} S\right)} f(x) d M u_{j} .
$$

Letting $j \rightarrow \infty$, from the weak convergence, (6.4), and (6.7) we get that $I I<\rho$ for $j$ sufficiently large. Combining all of these inequalities, we get (6.5). 
Now we prove (6.6). For $j \geq J_{0}$, we have $\bar{S}_{j} \subset \Omega_{0}$ and $\bar{S} \subset \Omega_{j}$. We estimate the difference as follows:

$$
\begin{aligned}
& \left|\int_{S_{j}} \delta\left(x, S_{j}\right)^{1-\epsilon} d M u_{j}-\int_{S} \delta(x, S)^{1-\epsilon} d M u_{0}\right| \\
& \leq\left|\int_{S_{j}} \delta\left(x, S_{j}\right)^{1-\epsilon} d M u_{j}-\int_{S} \delta(x, S)^{1-\epsilon} d M u_{j}\right| \\
& \quad+\left|\int_{S} \delta(x, S)^{1-\epsilon} d M u_{j}-\int_{S} \delta(x, S)^{1-\epsilon} d M u_{0}\right|=I+I I .
\end{aligned}
$$

The integral $I I$ goes to 0 by the weak convergence. To estimate $I$, we write

$$
\begin{aligned}
I & =\left|\int \chi_{S_{j}}(x) \delta\left(x, S_{j}\right)^{1-\epsilon} d M u_{j}-\int \chi_{S}(x) \delta(x, S)^{1-\epsilon} d M u_{j}\right| \\
& \equiv\left|\int f_{j}(x) d M u_{j}-\int f(x) d M u_{j}\right| \leq \int\left|f_{j}(x)-f(x)\right| d M u_{j} .
\end{aligned}
$$

We claim that $\left|f_{j}-f\right| \leq 1$ and $\left(f_{j}-f\right) \rightarrow 0$ pointwise a.e. (we define $\delta(x, S)=0$ for $x \notin \bar{S})$. The first assertion is trivial. If $x \in S$, then $\chi_{S_{j}}(x) \rightarrow \chi_{S}(x)=1$ by Lemma 5.5, and $\delta\left(x, S_{j}\right) \rightarrow \delta(x, S)$ by Lemma[5.7. If $x \notin \bar{S}$, then $x \notin S_{j}$ for large $j$; so $f_{j}(x)=f(x)=0$. This establishes the a.e. convergence. Let $A$ be a measurable set such that $S_{j}, S \subset A \Subset \Omega_{0}$. By Egorov's theorem, given $\delta>0$ there exists $E \subset A$ with $M u_{0}(E)<\delta$ and $f_{j}-f \rightarrow 0$ uniformly in $A \backslash E$. From (6.4) we have that $M u_{j}(A) \rightarrow M u_{0}(A)$ and $M u_{j}(E) \rightarrow M u_{0}(E)$ as $j \rightarrow \infty$, and so $M u_{j}(A) \leq 2 M u_{0}(A)$ and $M u_{j}(E)<2 \delta$ for $j$ sufficiently large. In addition, $\left|f_{j}(x)-f(x)\right|<\epsilon$ for all $x \in A \backslash E$ and all $j$ sufficiently large. Then

$$
\begin{aligned}
& \int\left|f_{j}(x)-f(x)\right| d M u_{j} \\
& =\int_{E}\left|f_{j}(x)-f(x)\right| d M u_{j}+\int_{A \backslash E}\left|f_{j}(x)-f(x)\right| d M u_{j} \\
& \leq M u_{j}(E)+\epsilon M u_{j}(A \backslash E) \leq 2 \delta+2 \epsilon M u_{0}(A),
\end{aligned}
$$

and hence $I \rightarrow 0$. Therefore, (6.6) follows, and Step 6 is complete.

Step 7. Now we will produce the point $x_{0}$ and the supporting hyperplane with the desired properties. By hypothesis,

$$
S_{j}=\left\{x \in \Omega_{j}: u_{j}(x)<l_{j}(x)+\frac{1}{j}\right\} \nsubseteq\left\{x \in \Omega_{j}: u_{j}(x)<-C \epsilon^{\prime}\right\}=T_{j} .
$$

Then there is a point $y_{j} \in S_{j} \cap T_{j}^{c}$. Then $u_{j}\left(y_{j}\right)<l_{j}\left(y_{j}\right)+\frac{1}{j}$, and we can assume $u_{j}\left(y_{j}\right)=-C \epsilon^{\prime}$. By (6.3) $), x_{j} \in \Omega_{0}$ and

$$
\operatorname{dist}\left(x_{j}, \partial \Omega_{0}\right) \geq \frac{\epsilon^{\prime}}{2} \quad \text { for all } j>j_{0}\left(\epsilon^{\prime}\right) .
$$

By Theorem 2.8 and Proposition 2.10,

$$
C \epsilon^{\prime n}=\left|u_{j}\left(y_{j}\right)\right|^{n} \leq C_{n} \delta\left(y_{j}, \Omega_{j}\right)^{\epsilon} \int_{\Omega_{j}} \delta\left(y, \Omega_{j}\right)^{1-\epsilon} d M u_{j} \leq C(n, \Lambda) \delta\left(y_{j}, \Omega_{j}\right)^{\epsilon} .
$$

This implies that $\operatorname{dist}\left(y_{j}, \partial \Omega_{j}\right) \geq C \epsilon^{\prime \frac{n}{\epsilon}}$. So by (6.3), $\operatorname{dist}\left(y_{j}, \Omega_{0}\right) \geq C\left(n, \epsilon^{\prime}, \Lambda\right)$ for $j$ large enough. 
Therefore, for all $j$ sufficiently large, $\left\{x_{j}\right\}$ and $\left\{y_{j}\right\}$ are contained in a compact subset of $\Omega_{0}$. By passing to subsequences, $x_{j} \rightarrow x_{0} \in \Omega_{0}$ and $y_{j} \rightarrow y_{0} \in \Omega_{0}$. Furthermore, $\operatorname{dist}\left(x_{0}, \partial \Omega_{0}\right), \operatorname{dist}\left(y_{0}, \partial \Omega_{0}\right) \geq C\left(\epsilon^{\prime}\right)$. This shows statement $(c)$ of the lemma. Let $p_{j} \in \mathbb{R}^{n}$ define $l_{j}(x)$, i.e., $l_{j}(x)=u_{j}\left(x_{j}\right)+p_{j} \cdot\left(x-x_{j}\right)$. Then since the $x_{j}$ are away from $\partial \Omega_{0}$, the $p_{j}$ are bounded (Step 3 ). Choose a subsequence so that $p_{j} \rightarrow p_{0}$. Now $u_{j}(x) \geq u_{j}\left(x_{j}\right)+p_{j} \cdot\left(x-x_{j}\right)=l_{j}(x)$ for all $x \in \Omega_{j}$. Let $j \rightarrow \infty$ to get $u_{0}(x) \geq u_{0}\left(x_{0}\right)+p_{0} \cdot\left(x-x_{0}\right)$ for all $x \in \Omega_{0}$. This means that $l_{0}(x)=u_{0}\left(x_{0}\right)+p_{0} \cdot\left(x-x_{0}\right)$ is a support plane to $u_{0}$ at $x_{0}$. Now $u_{j}\left(y_{j}\right)=-C \epsilon^{\prime}$ for all $j$, implying that $u_{0}\left(y_{0}\right)=-C \epsilon^{\prime}$. Also, $u_{j}\left(y_{j}\right) \leq l_{j}\left(y_{j}\right)+\frac{1}{j}$. Let $j \rightarrow \infty$ and get $u_{0}\left(y_{0}\right) \leq l_{0}\left(y_{0}\right)$, so that $u_{0}\left(y_{0}\right)=l_{0}\left(y_{0}\right)$. Therefore, $y_{0} \in S_{0} \cap T_{0}^{c}$. This proves $(d)$, and we are done.

We now turn to the second main result of this section. Since we now have in place the extremal points theorem and the selection lemma, the proof follows in much the same way as in [G01, Theorem 5.3.3]. The proof is by contradiction. If the statement is not true, there are a sequence of normalized domains $\Omega_{j}$ and a sequence of functions $u_{j}$ defined on those domains that satisfy the hypotheses of Lemma 6.1. The growth condition in Theorem 6.4 implies that the measures $M u_{j}$ are locally uniformly bounded.

Further, if Theorem 6.4 is not true, the additional hypotheses of Lemma 6.1 (those concerning $(c)$ and $(d)$ ) are also satisfied. As in [G01, Theorem 5.3.3], it is conclusion $(d)$ that produces the contradiction. This conclusion contradicts the extremal points theorem. For this result to apply, we need to know that $u_{0}$ is not identically zero. This is the reason for the assumption $0<\lambda \leq\left|\min _{\Omega_{j}} u_{j}\right|$ in Lemma 6.1.

Theorem 6.4. Given a convex, normalized domain $\Omega$ in $\mathbb{R}^{n}$, consider $u \in C(\bar{\Omega})$, convex, with $\left.u\right|_{\partial \Omega}=0$ and such that $M u \in D_{\epsilon_{1}}(C)$ and is absolutely continuous with respect to Lebesgue measure. Suppose that the density $d(x)$ of the measure $M u$ satisfies the growth condition: $d(x) \leq C_{1} \operatorname{dist}(K, \partial \Omega)^{-\beta}$ for each $K \subset \Omega$ compact and for a.e. $x \in K$, where $C_{1}$ and $\beta$ are positive constants; and $0<\lambda \leq\left|\inf _{\Omega} u\right|$.

Then for each $\epsilon>0$, there exists $\rho=\rho(\epsilon)$ such that for all $\Omega$ normalized, for all $x_{0}$ with $\operatorname{dist}\left(x_{0}, \partial \Omega\right) \geq \epsilon$, for all functions $u$ satisfying the above conditions, and for all supporting hyperplanes $l(x)$ to $u$ at $x_{0}$, we have that $\{x \in \Omega: u(x)<l(x)+\rho\}$ is compactly contained in $\Omega$.

Moreover, $\{x \in \Omega: u(x)<l(x)+\rho\} \subset\{x \in \Omega: u(x)<-\bar{C} \epsilon\}$, where $\bar{C}=$ $\bar{C}\left(C, \epsilon_{1}, n, \lambda\right)$ and $\rho$ depends only on $\epsilon, \epsilon_{1}, n, \lambda$, and the estimate on $d(x)$.

We conclude this section with an additional remark concerning the growth condition in Theorem 6.4 The $D_{\epsilon}$ condition imposes a constraint on the rate at which the measure can blow up near the boundary of the domain, as the following example illustrates.

Let $\Omega=B_{1}(0)$, let $u \in C(\bar{\Omega})$ be convex, and suppose $M u \in D_{\epsilon}$ and $M u=$ $f(x) d x$, where $f(x) \geq \operatorname{dist}(x, \partial \Omega)^{-\beta}$. Then by Lemma 5.9.

$$
\int_{B_{1}(0)} \delta\left(x, B_{1}(0)\right)^{1-\epsilon} f(x) d x \leq C \int_{B_{\frac{1}{2}}(0)} f(x) d x<\infty .
$$

Since $B_{1}(0)$ is normalized, the left-hand side of the last inequality is larger than $C \int_{B_{1}(0)} \operatorname{dist}\left(x, \partial B_{1}(0)\right)^{1-\epsilon} \operatorname{dist}\left(x, \partial B_{1}(0)\right)^{-\beta} d x$, which is not integrable for $\beta \geq$ $2-\epsilon$. 


\section{Hölder Continuity of the Gradient}

In order to obtain the interior $C^{1, \alpha}$ estimates we will use two technical lemmas concerning the relationship between the dilation of a normalized domain and the dilation of a sublevel set. We assume throughout this section that the function $u$ is not identically zero.

Lemma 7.1 (Compare with G01 Lemma 5.4.1]). Suppose $\Omega$ is a normalized convex domain, $u \in C(\bar{\Omega})$ is convex and $\left.u\right|_{\partial \Omega}=0, M u \in D_{\epsilon}(C)$ and is absolutely continuous with respect to Lebesgue measure and satisfies the growth condition in Theorem 6.4, and that $u\left(x_{0}\right)=\min _{\Omega} u<-\lambda<0$. Given $0<\eta \leq 1$, define

$$
\Omega_{\eta}=\left\{x \in \Omega: u(x)<(1-\eta) \min _{\Omega} u\right\} .
$$

Then there exists a constant $\nu, 0<\nu<1$, depending on $n, \epsilon$, the $D_{\epsilon}$ constant $C$, and $\lambda$, such that $\frac{1}{2} \Omega \subset \nu \Omega_{\frac{1}{2}}$, where the dilations are with respect to $x_{0}$.

The proof of Lemma 7.1] is nearly identical to the proof in [G01, and we omit it.

Lemma 7.2 (See G01, Corollary 5.4.4]). Suppose that $\Omega$ is a normalized convex domain, $u \in C(\bar{\Omega})$ is convex and $\left.u\right|_{\partial \Omega}=0, M u \in D_{\epsilon}(C)$ and is absolutely continuous with respect to Lebesgue measure and satisfies the growth condition in Theorem 6.4 and $u\left(x_{0}\right)=\min _{\Omega} u=-1$. Then there exists a constant $\nu \in(0,1)$ such that

$$
\Omega \subset(2 \nu)^{k} \Omega_{\frac{1}{2^{k}}}
$$

for $k=1,2,3, \ldots, \nu=\nu(n, \epsilon, C)$, where the dilations are with respect to $x_{0}$.

Proof. The case $k=1$ is covered by the previous lemma. The $\nu$ in the preceding result depends on $n, \epsilon$, the $D_{\epsilon}$ constant $C$, and $\min _{\Omega} u$. Since this minimum is -1 , in this case we can remove the dependence of $\nu$ on $\min _{\Omega} u$.

Now suppose $k=2$. Let $T_{1}$ normalize $\Omega_{\frac{1}{2}}$, and set $\Omega_{1}^{*}=T_{1}\left(\Omega_{\frac{1}{2}}\right)$. Let $v_{1}(x)=$ $2\left[u\left(T_{1}^{-1} x\right)+\frac{1}{2}\right]$. Then by Remark 2.7 $M v_{1} \in D_{\epsilon}(C)$, and we have that $\left.v_{1}\right|_{\partial \Omega_{1}^{*}}=0$ and $\min _{\Omega_{1}^{*}} v_{1}=v_{1}\left(T_{1} x_{0}\right)=-1$. Apply the previous lemma to $v_{1}$ in $\Omega_{1}^{*}$ to get $\frac{1}{2} \Omega_{1}^{*} \subset \nu\left(\Omega_{1}^{*}\right)_{\frac{1}{2}}$. Now

$$
\begin{aligned}
\left(\Omega_{1}^{*}\right)_{\frac{1}{2}} & =\left\{x \in \Omega_{1}^{*}: v_{1}(x)<\frac{1}{2} \min _{\Omega_{1}^{*}} v_{1}\right\}=\left\{x \in \Omega_{1}^{*}: 2\left[u\left(T_{1}^{-1} x\right)+\frac{1}{2}\right]<-\frac{1}{2}\right\} \\
& =\left\{x \in \Omega_{1}^{*}: u\left(T_{1}^{-1} x\right)<-\frac{3}{4}\right\}=T_{1}\left(\left\{x \in \Omega_{\frac{1}{2}}: u(x)<-\frac{3}{4}\right\}\right)=T_{1}\left(\Omega_{\frac{1}{4}}\right) .
\end{aligned}
$$

Therefore, $\nu\left(T_{1}\left(\Omega_{\frac{1}{4}}\right)\right)=T_{1}\left(\nu \Omega_{\frac{1}{4}}\right)$ contains $\frac{1}{2}\left(T_{1}\left(\Omega_{\frac{1}{2}}\right)\right)=T_{1}\left(\frac{1}{2} \Omega_{\frac{1}{2}}\right)$. Hence, by applying $T_{1}^{-1}$, we get $\frac{1}{2} \Omega_{\frac{1}{2}} \subset \nu \Omega_{\frac{1}{4}}$ or $\Omega_{\frac{1}{2}} \subset(2 \nu) \Omega_{\frac{1}{4}}$. Combining this with the preceding step, we get

$$
\Omega \subset(2 \nu) \Omega_{\frac{1}{2}} \subset(2 \nu)^{2} \Omega_{\frac{1}{4}} .
$$

In general, let $T_{k}$ normalize $\Omega_{\frac{1}{2^{k}}}$ and $v_{k}(x)=2^{k}\left[u\left(T_{k}^{-1} x\right)+\left(1-\frac{1}{2^{k}}\right)\right]$. As above, we conclude that $\Omega \frac{1}{2^{k-1}} \subset(2 \nu) \Omega_{\frac{1}{2^{k}}}$.

Theorem 7.3 (Compare with G01 Theorem 5.4.5]). Let $\Omega$ be bounded, open and convex, and let $u \in C(\bar{\Omega})$ be convex, with $\left.u\right|_{\partial \Omega}=0$. Then if $M u \in D_{\epsilon}(C)$ for some $\epsilon \in(0,1]$ and is absolutely continuous with respect to Lebesgue measure and satisfies the growth condition in Theorem 6.4, then $u$ is $C^{1, \alpha}$ in the interior of $\Omega$ for some $0<\alpha<1$. 
Proof. The proof proceeds in a sequence of steps.

Step 1. If $\Omega$ is normalized and $\min _{\Omega} u=u\left(x_{0}\right)=-1$, then $u$ is $C^{1, \alpha}$ at $x_{0}$.

The point $x_{0}$ where the minimum is attained is unique by Corollary 4.2. By Theorem 2.8 and Proposition 2.10 $\operatorname{dist}\left(x_{0}, \partial \Omega\right)>\rho$, where $\rho$ depends on $n, \epsilon$, and the $D_{\epsilon}$ constant; so $B_{\rho}\left(x_{0}\right) \subset \Omega$. Let $x \in \Omega, x \neq x_{0}$. Then there exists an integer $k \geq 1$ such that $2^{-k} \leq u(x)-u\left(x_{0}\right)<2^{-k+1}$. Then, since $u\left(x_{0}\right)=-1$, we get that $u(x) \geq-\left(1-2^{-k}\right) ;$ so $x \notin \Omega_{\frac{1}{2^{k}}}$. So by Lemma [7.2, $x \notin(2 \nu)^{-k} B_{\rho}\left(x_{0}\right)=$ $B \frac{\rho}{(2 \nu)^{k}}\left(x_{0}\right)$, and hence $\left|x-x_{0}\right| \geq \rho(2 \nu)^{-k}$. We can take $\nu>\frac{1}{2}$. So $\nu=2^{-\theta}$ for some $\theta \in(0,1)$. Hence $\left|x-x_{0}\right| \geq \rho\left(2^{-k}\right)^{1-\theta}$. Since $u(x)-u\left(x_{0}\right)<2^{-k+1}$, we see that $2^{-k}>\frac{u(x)-u\left(x_{0}\right)}{2}$. Therefore,

$$
\left|x-x_{0}\right| \geq \rho\left(2^{-k}\right)^{1-\theta}>\rho\left(\frac{u(x)-u\left(x_{0}\right)}{2}\right)^{1-\theta} .
$$

From this, we obtain $0 \leq u(x)-u\left(x_{0}\right) \leq 2\left(\frac{1}{\rho}\right)^{\frac{1}{1-\theta}}\left|x-x_{0}\right|^{\frac{1}{1-\theta}}$, proving the claim for this step, since $\frac{1}{1-\theta}>1$.

Step 2. If $\Omega$ is not necessarily normalized, and $\min _{\Omega} u$ is not necessarily -1 , then $u$ is $C^{1, \alpha}$ at its minimum $x_{0}$.

Let $T$ normalize $\Omega\left(T x=A x+b\right.$ for an invertible matrix $A$ and some $\left.b \in \mathbb{R}^{n}\right)$, and define $u^{*}(y)=\left|\min _{\Omega} u\right|^{-1} u\left(T^{-1} y\right)$. Then from Remark 2.7. $M u^{*} \in D_{\epsilon}(C)$ in $\Omega^{*}=T(\Omega)$ and $\min _{\Omega^{*}} u^{*}=-1$, and this minimum is attained at $T x_{0}$. Then by Step 1 we have that

$$
0 \leq u^{*}(y)-u^{*}\left(T x_{0}\right) \leq C(\epsilon, n, C)\left|y-T x_{0}\right|^{\alpha+1}
$$

Let $y=T x$. Then

or

$$
0 \leq u(x)-u\left(x_{0}\right) \leq C(\epsilon, n, C)\left|\min _{\Omega} u\right|\left|T x-T x_{0}\right|^{\alpha+1}
$$

$$
0 \leq u(x)-u\left(x_{0}\right) \leq C\|A\|^{\alpha+1}\left|\min _{\Omega} u\right|\left|x-x_{0}\right|^{\alpha+1} .
$$

Step 3. If $\Omega$ is normalized, then $u$ is $C^{1, \alpha}$ in the interior of $\Omega$.

We prove that if $\operatorname{dist}(\bar{x}, \partial \Omega) \geq \rho$, then

$$
\left|u(x)-l_{\bar{x}}(x)\right| \leq C\left(n, \epsilon, \rho, C,\left|\min _{\Omega} u\right|\right)|x-\bar{x}|^{\alpha+1},
$$

where $l_{\bar{x}}(x)$ is any support plane to $u$ at $\bar{x}$. By Theorem 6.4 there exists $\rho_{0}=$ $\rho_{0}\left(n, \epsilon, \rho, C,\left|\min _{\Omega} u\right|\right)$ such that

$$
\Omega_{\bar{x}, \rho_{0}} \equiv\left\{x \in \Omega: u(x)<l_{\bar{x}}(x)+\rho_{0}\right\} \subset\{x \in \Omega: u(x)<-\bar{C} \rho\},
$$

where $\bar{C}=\bar{C}\left(n, \epsilon, C,\left|\min _{\Omega} u\right|\right)$. Let $T$ normalize $\Omega_{\bar{x}, \rho_{0}}$ and let $v(x)=u(x)-l_{\bar{x}}(x)-$ $\rho_{0}$. Then $\left.v\right|_{\partial \Omega_{\bar{x}, \rho_{0}}}=0$ and $v(\bar{x})=\min _{\Omega_{\bar{x}, \rho_{0}}} v=-\rho_{0}$. Then, by Step 2,

$$
0 \leq v(x)-v(\bar{x}) \leq C(\epsilon, n, C)\left|\min _{\Omega_{\bar{x}, \rho_{0}}} v\right|\|A\|^{1+\alpha}|x-\bar{x}|^{1+\alpha}
$$

Then, since $\left|\min _{\Omega_{\bar{x}}, \rho_{0}} v\right|=\rho_{0}$, the claim holds if $\|A\|$ can be dominated independently of $\bar{x}$, in terms of the structure and $\rho$ only. As shown in [G01, p. 98], $\|A\|=\max \lambda_{i}^{-1}$, where the $\lambda_{i}$ are the lengths of the axes of the minimum ellipsoid of $\Omega_{\bar{x}, \rho_{0}}$ (see Theorem $\left.2.4(\mathrm{a})\right)$, and $\operatorname{det} A=\left(\lambda_{1}\right)^{-1} \cdots\left(\lambda_{n}\right)^{-1}$.

Define $u^{*}(x)=|\operatorname{det} A|^{\frac{2}{n}} v\left(T^{-1} x\right)$. We claim that $\left|\min _{T\left(\Omega_{\bar{x}, \rho_{0}}\right)} u^{*}\right|^{n}$ is comparable to $M u^{*}\left(\frac{1}{2} T\left(\Omega_{\bar{x}, \rho_{0}}\right)\right)$. Indeed, by Proposition 2.10 and the $D_{\epsilon}$ condition, we have that 
$\left|\min _{T\left(\Omega_{\bar{x}, \rho_{0}}\right)} u^{*}\right|^{n} \leq C \int_{T\left(\Omega_{\bar{x}, \rho_{0}}\right)} \delta\left(x, T\left(\Omega_{\bar{x}, \rho_{0}}\right)\right)^{1-\epsilon} d M u^{*} \leq M u^{*}\left(\frac{1}{2} T\left(\Omega_{\bar{x}, \rho_{0}}\right)\right)$. For the other inequality, we again use Proposition [2.10] and also use the fact that if $x \in$ $\frac{1}{2} T\left(\Omega_{\bar{x}, \rho_{0}}\right)$, then $\delta\left(x, T\left(\Omega_{\bar{x}, \rho_{0}}\right)\right) \geq C_{n}$. More precisely,

$$
\begin{aligned}
\left|\min _{T\left(\Omega_{\bar{x}, \rho_{0}}\right)} u^{*}\right|^{n} & \geq C \int_{T\left(\Omega_{\bar{x}, \rho_{0}}\right)} \delta\left(x, T\left(\Omega_{\bar{x}, \rho_{0}}\right)\right)^{1-\epsilon} d M u^{*} \\
& \geq C \int_{\frac{1}{2} T\left(\Omega_{\bar{x}, \rho_{0}}\right)} \delta\left(x, T\left(\Omega_{\bar{x}, \rho_{0}}\right)\right)^{1-\epsilon} d M u^{*} \geq C M u^{*}\left(\frac{1}{2} T\left(\Omega_{\bar{x}, \rho_{0}}\right)\right) .
\end{aligned}
$$

We have that $\left|\min _{T\left(\Omega_{\bar{x}, \rho_{0}}\right)} u^{*}\right|^{n}=|\operatorname{det} A|^{2} \rho_{0}^{n}$ and (by (2.1)

$$
M u^{*}\left(\frac{1}{2} T\left(\Omega_{\bar{x}, \rho_{0}}\right)\right)=|\operatorname{det} A| M u\left(T^{-1}\left(\frac{1}{2} T\left(\Omega_{\bar{x}, \rho_{0}}\right)\right)\right) .
$$

This implies that

$$
|\operatorname{det} A|^{2} \rho_{0}^{n} \approx|\operatorname{det} A| M u\left(T^{-1}\left(\frac{1}{2} T\left(\Omega_{\bar{x}, \rho_{0}}\right)\right)\right)
$$

or

$$
|\operatorname{det} A| \leq C \rho_{0}^{-n} M u\left(T^{-1}\left(\frac{1}{2} T\left(\Omega_{\bar{x}, \rho_{0}}\right)\right)\right) \leq C \rho_{0}^{-n} M u(\{x \in \Omega: u(x)<-\bar{C} \rho\}) .
$$

Since $\lambda_{i} \leq 1,\|A\|=\max \left\{\lambda_{i}^{-1}\right\}=\lambda_{j}^{-1} \leq\left(\lambda_{1}\right)^{-1} \cdots\left(\lambda_{n}\right)^{-1}=\operatorname{det} A$. Therefore $\|A\|$ can be estimated by $n, \epsilon$, the $D_{\epsilon}$ constant, $\rho,|\min u|$ and $M u(\{x \in \Omega: u(x)<$ $-\bar{C} \rho\})$. This establishes the claim for this step.

Step 4. If $\Omega$ is not normalized, then $u$ is $C^{1, \alpha}$ in the interior of $\Omega$.

Let $T$ be an affine transformation that normalizes $\Omega$, and define $u^{*}(y)=u\left(T^{-1} y\right)$ for $y \in T(\Omega)$. Now apply Step 3 to $u^{*}$ in the normalized domain $T(\Omega)$. The constant appearing in the inequality will also depend on $\|T\|$, which depends on the eccentricity and volume of $\Omega$.

\section{REFERENCES}

[C90] Caffarelli, L. A. 1990. A localization property of viscosity solutions to the Monge-Ampère equation and their strict convexity. Ann. of Math. 131, 129-134. MR 91f:35058

[C91] Caffarelli, L. A. 1991. Some regularity properties of solutions of Monge-Ampère equation. Comm. Pure Appl. Math. 44, 965-969. MR 92h:35088

[G01] Gutiérrez, C. E. 2001. The Monge-Ampère Equation. Birkhaüser, Boston, MA. MR 2002e:35075

[GH00] Gutiérrez, C. E. and Qingbo Huang 2000. Geometric properties of the sections of solutions to the Monge-Ampère equation. Trans. Amer. Math. Soc. 352, 4381-4396. MR 2000m:35060

[J92] Jerison, D. 1992. Sharp estimates for harmonic measure in convex domains. In "Partial differential equations with minimal smoothness and applications," IMA Volumes in Mathematics and its Applications, vol. 42, edited by B. Dalhberg et al., Springer-Verlag, 149-162. MR 93d:31004

[J96] Jerison, D. 1996. A Minkowski problem for electrostatic capacity, Acta Math. 176 : 1-47. MR 97e:31003

[P78] Pogorelov, A. V. 1978. The Minkowski Multidimensional Problem. V. H. Winston and Sons, Washington, D.C. MR 57:17572

[Sc93] Schneider, R. 1993. Convex Bodies: The Brunn-Minkowski Theory. Cambridge University Press, Cambridge. MR 94d:52007 
[St93] Stein, E. M. 1993. Harmonic Analysis: Real-Variable Methods, Orthogonality, and Oscillatory Integrals. Princeton Mathematical Series, No. 43, Monographs in Harmonic Analysis, III, Princeton University Press, Princeton, NJ. MR 95c:42002

[TW97] Trudinger, N. S. and Wang, X-J. 1997. Hessian measures I. Topological Methods in Nonlinear Analysis 10, 225-239. MR 2000a:35061

Department of Mathematics, Temple University, Philadelphia, Pennsylvania 19122

E-mail address: gutierrez@math.temple.edu

Department of Mathematics, University of Utah, Salt Lake City, Utah 84112

E-mail address: hartenst@math.utah.edu 